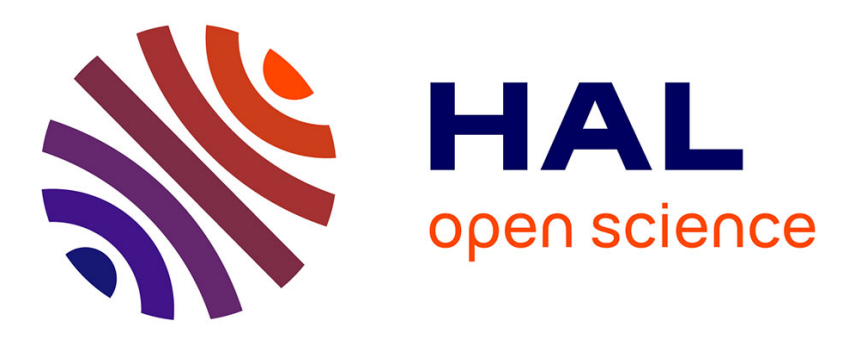

\title{
Homogenized magneto-elastic behavior model for the computation of strain due to magnetostriction in transformers
}

Mingyong Liu, Olivier Hubert, Xavier Mininger, Frédéric Bouillault, Laurent Bernard

\section{To cite this version:}

Mingyong Liu, Olivier Hubert, Xavier Mininger, Frédéric Bouillault, Laurent Bernard. Homogenized magneto-elastic behavior model for the computation of strain due to magnetostriction in transformers. IEEE Transactions on Magnetics, 2016, 52 (2), pp.8000212. 10.1109/TMAG.2015.2493062 . hal01244934

\section{HAL Id: hal-01244934 \\ https://hal-centralesupelec.archives-ouvertes.fr/hal-01244934}

Submitted on 17 Mar 2020

HAL is a multi-disciplinary open access archive for the deposit and dissemination of scientific research documents, whether they are published or not. The documents may come from teaching and research institutions in France or abroad, or from public or private research centers.
L'archive ouverte pluridisciplinaire HAL, est destinée au dépôt et à la diffusion de documents scientifiques de niveau recherche, publiés ou non, émanant des établissements d'enseignement et de recherche français ou étrangers, des laboratoires publics ou privés. 


\title{
Homogenized magneto-elastic behavior model for the computation of strain due to magnetostriction in transformers
}

\author{
Mingyong Liu ${ }^{1,2}$, Olivier Hubert ${ }^{2}$, Xavier Mininger ${ }^{1}$, Frédéric Bouillault ${ }^{1}$, and Laurent Bernard ${ }^{1}$ \\ ${ }^{1}$ GeePs, CNRS UMR 8507, CentraleSupelec, Univ Paris Sud, UPMC \\ 11 rue Joliot-Curie 91192 Gif-sur-Yvette, France \\ ${ }^{2}$ LMT-Cachan, CNRS UMR 8535, ENS-Cachan, Univ. Paris-Saclay \\ 61 Av. du Président Wilson 94235 Cachan Cedex, France
}

\begin{abstract}
This paper deals with the prediction of the deformation of a multi-layer transformer core made of an assembly of anisotropic 'E-shaped' and 'I-shaped' sheets. This magneto-mechanical coupled problem is solved by a stepping finite element method sequential approach: magnetic resolution is followed by mechanical resolution. A 3D Simplified Multi-Scale Model (SMSM) describing both magnetic and magnetostrictive anisotropies is used as the constitutive law of the material. The transformer core structure is modeled in 2D and an homogenization technique is implemented to take the anisotropic behavior of each layer into consideration and define an average behavior at each element of the finite element mesh. A three-layers transformer prototype is fabricated with Hi-B grain-oriented iron-silicon alloy. Experimental measurements are carried out and compared to the modeling results. Small discrepancies are observed and discussed.
\end{abstract}

\section{Index Terms}

Magnetostriction, transformers, multiscale modeling, iron-silicon alloys, finite element method.

\section{INTRODUCTION}

$\mathbf{T}$ HE Aeronautic world is undergoing deep changes associated with the increase of on-board electrical equipments. The electrical power supplied by the generators plugged to the turbojet has to be increased to feed these systems. This power is commonly transformed through power electronic devices and transformers to adapt voltage, current and frequency to the final on board user. Therefore the increase of the electrical power leads to an increase of the size and mass of these devices at constant power to mass ratio.

The use of magnetic materials presenting a higher power density (e.g. iron-cobalt) could be a solution to reduce this supplementary mass of 3-phases transformers. Such prototypes generate unfortunately a loud noise in operation caused by the interaction between the transformer's magnetic stray field and the current-carrying winding loops [1] and also by periodic deformations of sheets linked with the structure of the transformer core [2] [3]. This deformation has two origins: i) elastic strain associated to magnetic forces appearing on the free surface and volume; ii) spontaneous magnetostriction depending on the local magnetic state of the material [4]. Magnetic forces, calculated by the virtual work method [5], are induced by the field on a medium with inhomogeneous permeability. They are usually considered negligible in transformer structures due to small flux leakage (excitation below the magnetization saturation of sheets and small air gaps). Magnetostrictive strain is associated with the re-organization of magnetic domains that usually subdivide each grain of a polycrystal. They are characterized by a magnetization vector $\vec{M}$ whose magnitude equals the saturation magnetization of the material, and free magnetostriction strain $\epsilon_{\mu}$ depending on the magnetostriction constants and magnetization direction. The latter is usually associated to some specific crystallographic direction depending on the magneto crystalline anisotropy. 
When a magnetic field $\vec{H}$ is applied, magnetization vectors rotate toward the direction of the applied field leading to a displacement of domain walls separating magnetic domains, increasing the volume fraction of domains aligned with the field. Thus a deformation appears at the macroscopic scale which is induced by the free strain $\boldsymbol{\epsilon}_{\mu}$ of the considered domains. The crystallographic texture has a strong impact on the magnetostrictive behavior [6], which in our case leads to magnetic and magnetostrictive anisotropies (coupled magneto-mechanical phenomena with isotropic magnetic and magnetostrictive behaviors are studied in [7] [8]).

In this work, a complete chain from the material and external loading to the prediction of the transformer deformation is presented. It involves: i) a multiscale constitutive law able to give an accurate prediction of local magneto-mechanical behavior and fast enough to allow its implementation in a finite element modeling; ii) an homogenization procedure to reduce the $3 \mathrm{D}$ real problem in a $2 \mathrm{D}$ finite element modeling; ii) a finite element modeling indicating the prediction of displacement fields. A comparison to experimental results using Grain-Oriented (GO) silicon steel sheets is finally proposed. Indeed this material exhibits a very well known and understood anisotropy and a high level of magnetostriction along the transversal direction (TD) perpendicular to the rolling direction (RD) [6]. This behavior, the structure geometry and the boundary conditions generate some specific deformation harmonics measured thanks to a dedicated benchmark. Modeling results are in accordance with experimental data, but small discrepancies are observed and discussed

\section{THREE-PHASE TRANSFORMER AND STATE OF THE ART}

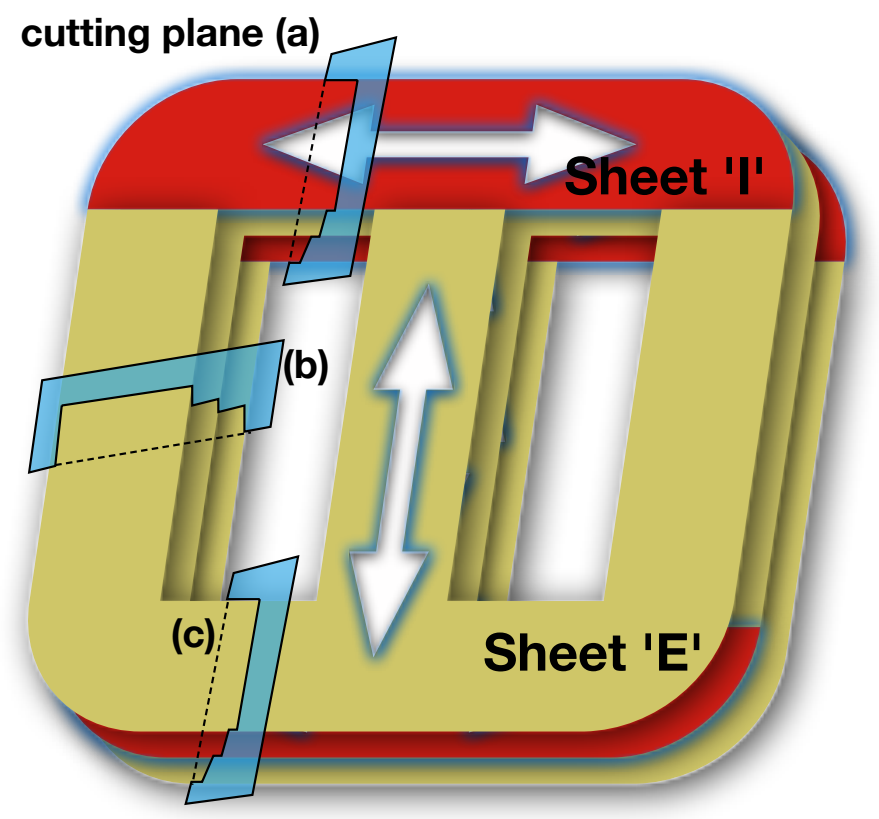

Fig. 1. Transformer core structure : 'E-shaped' sheet (yellow region) + 'I-shaped' sheet (red region) with indication of RD (white arrows). $\mathrm{RD}$ of the 'E-shaped' sheet is vertical and RD of the 'I-shaped' sheet is horizontal.

A three-phase transformer is made of three sets of primary and secondary windings wounded around the three legs of the transformer. The transformer core is usually made of very soft ferromagnetic materials for higher efficiency. The transformer core is made of an assembly of hundreds of thin sheets to limit the eddy current and ensure a homogeneous magnetic field through the thickness. Associated to their forming process (hot/cold rolling, heat treatments), transformer sheets usually exhibit anisotropic magnetic behaviors. Classical on-board electrical transformers are for example made of Non-Oriented FeSi or FeCo alloys that exhibit the highest induction at a given magnetic field level along the rolling direction (RD). The transformer core is consequently designed to increase the volume of material offering improved 
magnetic properties (namely permeability) in the direction of magnetic field. Since the transformer core is the result of an assembly of sheets in form of 'E-shaped' and 'I-shaped' for winding facility, 'E-shaped' and 'I-shaped' sheets are cut along the rolling direction of the lamination as shown in Fig.1 (white arrows indicate the RD). They are positioned alternatively on top or on bottom of the transformer in order to limit the parasitic air-gap [9]. This point is illustrated in figure 1 where red and yellow parts indicate 'I-shaped' and 'E-shaped' sheet respectively in case of a three layers transformer. Layers are composed of two families, family 1 and family 2 hereafter. (Family 1 corresponds to the layers with 'I-shaped' sheet on top and 'E-shaped' sheet on bottom. Family 2 corresponds to the layers with 'I-shaped' sheet on bottom and 'E-shaped' sheet on top.) Sectional views in different regions of this transformer are presented in figure 2. The sectional surface of sheet in regions (a), (b) and (c) are composed of $\{2 / 3 \mathrm{RD}+1 / 3$ $\mathrm{TD}\},\{1 / 1 \mathrm{RD}\}$ and $\{1 / 3 \mathrm{RD}+2 / 3 \mathrm{TD}\}$ respectively, leading to some variations of the average along the magnetic circuit.

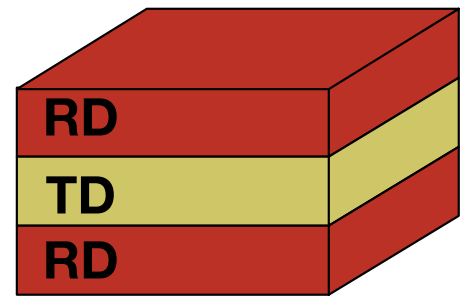

(a)

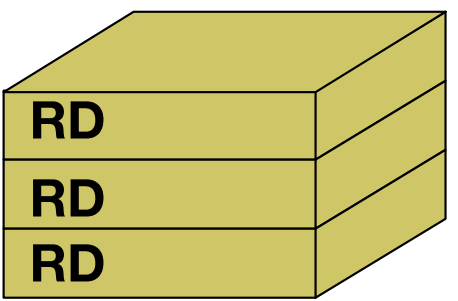

(b)

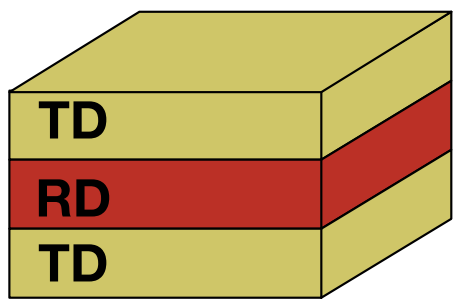

(c)

Fig. 2. Sectional view of the transformer prototype in different regions.

The noise generated by transformers has been studied for several decades and the related literature is abundant. Transformers generating noise can be separated into load noise [1] [10] and no-load noise. The former is due to magnetic interactions (especially Lorenz force) between the current carrying windings and transformer's magnetic stray field. The latter is caused by core vibrations which are more complex resulting from many phenomena. Up to now, several factors have been claimed to have relevance to the flux distribution and core vibrations, such as bolt holes [11], core clamping [12] and core structures [2]. Many works have been done at several levels:

- Electrical steel sheet level: Recently, magnetostriction of these electrical sheets under rotational magnetization has been characterised in [13] [14] and modeled in [15], giving better understanding of the magneto-mechanical behavior in T-joint. Modeling of transformer laminations with hysteresis loop in direction RD and TD with or without applied stress have been studied in [16] [17]. These works bring fundamental information for the precise modeling of transformers' dynamic behavior.

- Laminated structure level: With a set of electrical steel sheets, an early study shows that the magnetic flux transfers from lamination to lamination during the magnetization process [18]. Recent proposed homogeneous models, taking account of the laminated structure is able to express the flux density, force density, displacement distributions of the real laminated core [19] and more precisely the equivalent permeability of the over-lap joints [20]. But these are limited to an isotropic in-plane magnetic behaviour. A study of in-plane and out-of-plane vibrations is carried out using a set of electrical steel sheets [21], which reveals the importance of research on the cross-axis transmission of vibrations.

- Entier transformer: Those early relevance studies reveal the relevance of magnetostriction and magnetostatic forces to the vibration and noise of transformer cores [9] [22] [23]. Precise measurement of the magnetic flux in the transformer core [24] and thourough spectral analysis of magnetostriction induced forces [26] have also been studied. Although there are various works related to the estimation of the transformers' noise emission, rare literature is concerning a complete modeling chain of an entire transformer with laminated structure, from material characterization to the estimation of core vibration. 
Besides, several techniques exist to reduce the core vibrations. Step-lap design of the joints [27] in a transformer core is known to reduce core noise because of the better distribution of magnetic flux. Also, attempts are made to reduce the air gap between the lamination in order to eliminate the magnetic force in plan and off plan. This can be done by filling the air gap with nanocrystalline soft magnetic composite material (NSMC) with high permeability [28].

In this paper, only in-plane core vibration is considered which generates part of the no-load noise. Modelling is carried out under hypotheses of no statical nor dynamic losses. Magnetostrictive behaviors under rotational induction are not taken into concern. All these simplification are made to make the calculation possible to carried out in a personal computer within a reasonable time.

\section{Constitutive LAW}

\section{A. Multi-Scale Model}

A constitutive law of the magnetic material is first required for the modeling. A simplified version of a full multi-scale model (MSM) [29][6] is used. It involves a simplification of the description of a polycrystal through an equivalent single crystal that will exhibit approximately the same anisotropies, magnetic and magnetostrictive behaviors as the polycrystal. Using this simplification, the degrees of freedom are reduced, so that the whole numerical process can be carried out with a personal computer. In this simplified model, the magnetic material is described as a set of magnetic domains $\alpha$ with saturation magnetization $M_{s}$ and random orientation $\vec{\gamma}^{\alpha}\left(\vec{M}^{\alpha}=M_{s} \vec{\gamma}^{\alpha}\right)$.

The local free energy of a magnetic domain $W_{\text {tot }}^{\alpha}$ is expressed as the sum of four contributions (1): the magneto-static energy $W_{m a g}^{\alpha}$, the magneto-crystalline energy $W_{a n}^{\alpha}$, the configuration energy $W_{c o n f}^{\alpha}$ and the magneto-elastic energy $W_{m e}^{\alpha}$.

$$
W_{t o t}^{\alpha}=W_{m a g}^{\alpha}+W_{a n}^{\alpha}+W_{c o n f}^{\alpha}+W_{m e}^{\alpha}
$$

$W_{\text {mag }}^{\alpha}$ (equation (2) - where $\mu_{0}$ is the vacuum permeability) tends to align the local magnetization $\vec{M}^{\alpha}$ along the magnetic field $\vec{H}$ considered as homogeneous over the crystal.

$$
W_{m a g}^{\alpha}=-\mu_{0} \vec{M}^{\alpha} \cdot \vec{H}
$$

$W_{a n}^{\alpha}$ (equation (3)) tends to align the magnetization along the easy axes, that explains the existence of domain microstructure. In the form proposed here, $K_{1}$ is the so-called magneto crystalline energy constant. $\mathbb{P}$ is a $4^{\text {th }}$ order tensor that describes the magneto-crystalline anisotropy in the crystal frame $(\mathrm{CF}$ - $(x y z)) . \boldsymbol{Q}$ is a simple transformation matrix from CF to the sample frame (SF - $(X Y Z)$ ). ${ }^{t} \boldsymbol{Q}$ denotes its transposed form. The combination of the transformation matrix with the anisotropy matrix allows the expression of a large variety of anisotropies at the macroscale. Several definitions of $\mathbb{P}$ (using a $6 \times 6$ Voigt representation) are given in equation (4) for cubic symmetry (a), uniaxial symmetry of axis $x$ (b) and isotropy (c).

$$
\begin{gathered}
W_{a n}^{\alpha}=K_{1}\left(\vec{\gamma}^{\alpha} \otimes \vec{\gamma}^{\alpha}\right):\left(\boldsymbol{Q} \cdot \boldsymbol{Q} \cdot \mathbb{P} \cdot{ }^{t} \boldsymbol{Q} \cdot{ }^{t} \boldsymbol{Q}\right):\left(\vec{\gamma}^{\alpha} \otimes \vec{\gamma}^{\alpha}\right) \\
(a) \quad \mathbb{P}=\frac{1}{3}\left(\begin{array}{lll:lll}
0 & 1 & 1 & 0 & 0 & 0 \\
1 & 0 & 1 & 0 & 0 & 0 \\
1 & 1 & 0 & 0 & 0 & 0 \\
0 & 0 & 0 & 1 & 0 & 0 \\
0 & 0 & 0 & 0 & 1 & 0 \\
0 & 0 & 0 & 0 & 0 & 1
\end{array}\right)_{C F} \quad\left(\text { (b) } \quad \mathbb{P}=\frac{1}{3}\left(\begin{array}{llllll}
0 & 1 & 1 & 0 & 0 & 0 \\
1 & 3 & 2 & 0 & 0 & 0 \\
1 & 2 & 3 & 0 & 0 & 0 \\
0 & 0 & 0 & 1 & 0 & 0 \\
0 & 0 & 0 & 0 & 2 & 0 \\
0 & 0 & 0 & 0 & 0 & 1
\end{array}\right)_{C F} \quad(c) \quad \mathbb{P}=\frac{1}{3}\left(\begin{array}{cccccccc}
3 & 2 & 2 & 0 & 0 & 0 \\
2 & 3 & 2 & 0 & 0 & 0 \\
2 & 2 & 3 & 0 & 0 & 0 \\
0 & 0 & 0 & 2 & 0 & 0 \\
0 & 0 & 0 & 0 & 2 & 0 \\
0 & 0 & 0 & 0 & 0 & 2
\end{array}\right)\right.
\end{gathered}
$$


$W_{m e}^{\alpha}$ (equation (5)) is the magneto-elastic energy. It is written as a function of the free magnetostriction strain $\boldsymbol{\epsilon}_{\mu}^{\alpha}$ (given by equation (6) in the $\mathrm{CF}$ and equation (7) in the SF) and of the stress tensor $\sigma$ over the crystal. $\gamma_{i}$ are the direction cosines of the magnetization vector in CF and $\lambda_{100}$ (resp. $\lambda_{111}$ ) is the saturation magnetostriction strain along the direction $<100>$ (resp. $<111>$ ) of the single crystal. A stronger simplification (regarding for example the definition of a unique magnetostrictive constant $\lambda_{100}=$ $\lambda_{111}=\lambda_{s}$ ) is possible to describe quasi isotropic polycrystal thanks to the simplified MSM [16]. $W_{\text {con } f}^{\alpha}$ is a configuration term that accounts for the possible non randomness of the initial domain configuration (in the absence of applied magneto-mechanical loading) due for instance to plastic deformation [30] or to surface (demagnetizing) effects [6]. $C$ is a constant that defines the maximum level of the configuration energy. $N$ matrix allows the description of initial dissymmetries in the domains distribution. It is usually defined as a normalized diagonal matrix in the sample frame (9). In case of macroscopic demagnetizing effects, $N$ can be considered as a so-called form effect matrix [6]. $N_{i i}$ varies from zero to one, in relation with the sample geometry.

$$
\begin{gathered}
W_{m e}^{\alpha}=-\boldsymbol{\sigma}: \boldsymbol{\epsilon}_{\mu}^{\alpha} \\
\boldsymbol{\epsilon}_{\mu C F}^{\alpha}=\frac{3}{2}\left(\begin{array}{ccc}
\lambda_{100}\left(\gamma_{1}^{2}-\frac{1}{3}\right) & \lambda_{111} \gamma_{1} \gamma_{2} & \lambda_{111} \gamma_{1} \gamma_{3} \\
\lambda_{111} \gamma_{1} \gamma_{2} & \lambda_{100}\left(\gamma_{2}^{2}-\frac{1}{3}\right) & \lambda_{111} \gamma_{2} \gamma_{3} \\
\lambda_{111} \gamma_{1} \gamma_{3} & \lambda_{111} \gamma_{2} \gamma_{3} & \lambda_{100}\left(\gamma_{3}^{2}-\frac{1}{3}\right)
\end{array}\right)_{C F} \\
\boldsymbol{\epsilon}_{\mu}^{\alpha}=\boldsymbol{Q} \cdot \boldsymbol{\epsilon}_{\mu C F}^{\alpha} \cdot{ }^{t} \boldsymbol{Q} \\
W_{\text {conf }}^{\alpha}=C^{t} \vec{\gamma}^{\alpha} \cdot \boldsymbol{N} \cdot \vec{\gamma}^{\alpha} \\
\boldsymbol{N}=\left(\begin{array}{ccc}
N_{x x} & 0 & 0 \\
0 & N_{y y} & 0 \\
0 & 0 & N_{z z}
\end{array}\right)_{S F}
\end{gathered}
$$

Once the free energy is known for a given domain $\alpha$ of direction $\vec{\gamma}^{\alpha}$, its volume fraction $f_{\alpha}$ is calculated according to an explicit Boltzmann-type relation (10) [31], [29].

$$
f_{\alpha}=\frac{\exp \left(-A_{s} W^{\alpha}\right)}{\int_{\alpha} \exp \left(-A_{s} W^{\alpha}\right)}
$$

$A_{s}$ is an adjusting parameter related to the initial magnetic susceptibility $\chi_{0}$ of the material in absence of external or configuration loading (11).

$$
A_{s}=\frac{3 \chi_{0}}{\mu_{0} M_{s}^{2}}
$$

The magnetic and magnetostrictive behaviors of polycrystal are defined as the average values of local quantities (12)(13).

$$
\begin{aligned}
\vec{M} & =\int_{\alpha} f_{\alpha} \vec{M}^{\alpha} \\
\boldsymbol{\epsilon}_{\mu} & =\int_{\alpha} f_{\alpha} \boldsymbol{\epsilon}_{\mu}^{\alpha}
\end{aligned}
$$

The simplified MSM gives finally the average magnetostriction strain and magnetization at a given magnetic field $\vec{H}$ and stress $\sigma$. 


\section{B. Magnetic and Mechanical Homogenization of Two Media}

The calculation in reasonable time requires to use a $2 \mathrm{D}$ description of the transformer structure $((X, Y)$ plane). Figure 2 shows clearly that the reduction of the 3D structure into $2 \mathrm{D}$ must take the disposition and behavior of each layer into account. An homogenization strategy of a heterogeneous problem is required to extract the average behavior from the behavior of each family of layer. The following assumptions are considered:

- Sheets are normally very thin, allowing to assume a 2D homogeneous magnetic field using the classical condition of tangential magnetic field continuity. $\vec{H}_{1}$ and $\vec{H}_{2}$ denoting the magnetic field in each family, the following relation is obtained (14).

$$
\vec{H}_{1}=\vec{H}_{2}=\vec{H}
$$

- Sheets are normally very thin and considered as perfectly stuck together, allowing to assume a homogeneous displacement field at the interface between two sheets. Homogeneous strain can consequently be considered in the sheet plane. $\epsilon_{1}$ and $\epsilon_{2}$ denoting the total deformation in each family, the homogeneous strain hypothesis within the sheet plane $(X Y)$ leads to:

$$
\boldsymbol{\epsilon}_{1 X Y}=\boldsymbol{\epsilon}_{2 X Y}=\boldsymbol{\epsilon}_{X Y}
$$

$\epsilon$ indicates the average deformation.

- The transformer is supposed mechanically unloaded and thin enough to consider stress free conditions on upper and lower surfaces. This assumption leads to:

$$
\sigma_{1} \cdot \vec{Z}=\sigma_{2} \cdot \vec{Z}=\boldsymbol{\sigma} \cdot \vec{Z}=\overrightarrow{0}
$$

$\sigma$ indicates the average stress.

- Elastic and magnetostrictive deformations are considered sufficiently small to allow additive description of total deformation:

$$
\boldsymbol{\epsilon}=\boldsymbol{\epsilon}_{\mu}+\boldsymbol{\epsilon}_{e}
$$

$\boldsymbol{\epsilon}_{e}$ indicates the elastic strain tensor.

The magnetic flux $\phi$ circulating in the transformer through the surface $S$ of normal $\vec{n}$ belonging to the sheet plane can be expressed as sum of the flux in families 1 and 2 of sections $S_{1}$ and $S_{2}$ of normal $\vec{n}$ (18).

$$
\phi=\iint_{S} \vec{B} \cdot \vec{n} d s=\iint_{S_{1}} \overrightarrow{B_{1}} \cdot \vec{n} d s+\iint_{S_{2}} \vec{B}_{2} \cdot \vec{n} d s
$$

At a given point of the $2 \mathrm{D}$ problem, induction is homogeneous through the thickness for each family since magnetic field is homogeneous as well. The flux conservation relation is rewritten in:

$$
\vec{B}=f_{1} \vec{B}_{1}+f_{2} \vec{B}_{2}
$$

with $f_{1}$ and $f_{2}$ the section (or volume) fraction of family 1 and 2 respectively $\left(f_{1}+f_{2}=1\right.$ ). This mixing rule applies to the magnetization as well:

$$
\vec{M}=f_{1} \vec{M}_{1}+f_{2} \vec{M}_{2}
$$

$\vec{M}$ is the homogenized magnetization.

The mechanical resultant $\vec{R}$ over a surface $S$ of normal $\vec{n}$ belonging to the sheet plane can be expressed as sum of the mechanical resultant in families 1 and 2 of sections $S_{1}$ and $S_{2}$ of normal $\vec{n}$ as function of the average stress $\boldsymbol{\sigma}$ and the stress in each medium following: 


$$
\vec{R}=\iint_{S} \boldsymbol{\sigma} \cdot \vec{n} d s=\iint_{S_{1}} \boldsymbol{\sigma}_{1} \cdot \vec{n} d s+\iint_{S_{2}} \boldsymbol{\sigma}_{2} \cdot \vec{n} d s
$$

At a given point of the 2D problem, the stress is homogeneous through the thickness for each family since deformation (especially elastic) is homogeneous as well. The resultant conservation relation is rewritten in:

$$
\boldsymbol{\sigma}=f_{1} \boldsymbol{\sigma}_{1}+f_{2} \boldsymbol{\sigma}_{2}
$$

As underlined before, the total strain $\epsilon$ is the sum of elastic strain $\epsilon_{e}$ and magnetostrictive strain $\epsilon_{\mu}$. Applying Hooke's law to each family the following expressions of local stress tensor are obtained:

$$
\begin{aligned}
& \boldsymbol{\sigma}_{1}=\mathbb{C}_{1}: \boldsymbol{\epsilon}_{e 1}=\mathbb{C}_{1}:\left(\boldsymbol{\epsilon}_{1}-\boldsymbol{\epsilon}_{\mu 1}\right) \\
& \boldsymbol{\sigma}_{2}=\mathbb{C}_{2}: \boldsymbol{\epsilon}_{e 2}=\mathbb{C}_{2}:\left(\boldsymbol{\epsilon}_{2}-\boldsymbol{\epsilon}_{\mu 2}\right)
\end{aligned}
$$

$\mathbb{C}_{1}$ and $\mathbb{C}_{2}$ are the $4^{\text {th }}$ order stiffness tensor of families 1 and 2 . The average stress is on the other hand related to the average elastic strain using the effective stiffness tensor $\mathbb{C}$.

$$
\boldsymbol{\sigma}=\mathbb{C}: \boldsymbol{\epsilon}_{e}=\mathbb{C}:\left(\boldsymbol{\epsilon}-\boldsymbol{\epsilon}_{\mu}\right)
$$

The mixing rule (22) is applied and simplified using the homogeneous deformation hypothesis, leading to:

$$
\mathbb{C}=f_{1} \mathbb{C}_{1}+f_{2} \mathbb{C}_{2}
$$

on the one hand (usual averaging operation over the stiffness tensor - Voigt bound), and:

$$
\boldsymbol{\epsilon}_{\mu}=f_{1}\left(\mathbb{C}^{-1} \mathbb{C}_{1}\right): \boldsymbol{\epsilon}_{\mu 1}+f_{2}\left(\mathbb{C}^{-1} \mathbb{C}_{2}\right): \boldsymbol{\epsilon}_{\mu 2}
$$

on the other hand. This new mixing rule over the magnetostriction strain allows the calculation of a homogenized magnetostriction deformation depending on the stiffness, volume fraction and magnetostriction strain of each layer.

\section{NUMERICAL IMPLEMENTATION}

The coupling between magnetic and mechanical problems is considered through the magnetostriction that induces a loading of the mechanical problem. For simplicity reasons, the following supplementary simplifications have been made:

- Airgaps are not considered in the numerical modeling. The effect of magnetic forces is supposed of second order, and is verified by a numeric calculation (Fig. 12).

- Since magnetostrictive strain is relatively small, the stress level (local and global) is considered as small enough to have a negligible effect on the behaviors, which means that magnetostrictive strain only depends on applied magnetic field.

- Stiffness is considered as isotropic depending only on Young's modulus $E$ and Poisson's ratio $\nu$, leading to an homogeneous mechanical behavior over the layer families, which strongly simplifies the the magnetostriction mixing rule:

$$
\boldsymbol{\epsilon}_{\mu}=f_{1} \boldsymbol{\epsilon}_{\mu 1}+f_{2} \boldsymbol{\epsilon}_{\mu 2}
$$

- A single phase sinusoidal current excitation is considered in the following. Extension to a three-phases excitation and imposed flux is discussed in [32].

A sequential resolution of the problem is implemented: the magnetic resolution is followed by the mechanical resolution leading to a numerical weak coupling. This point is summed up in figure 3 and the algorithm is given in figure 4. 


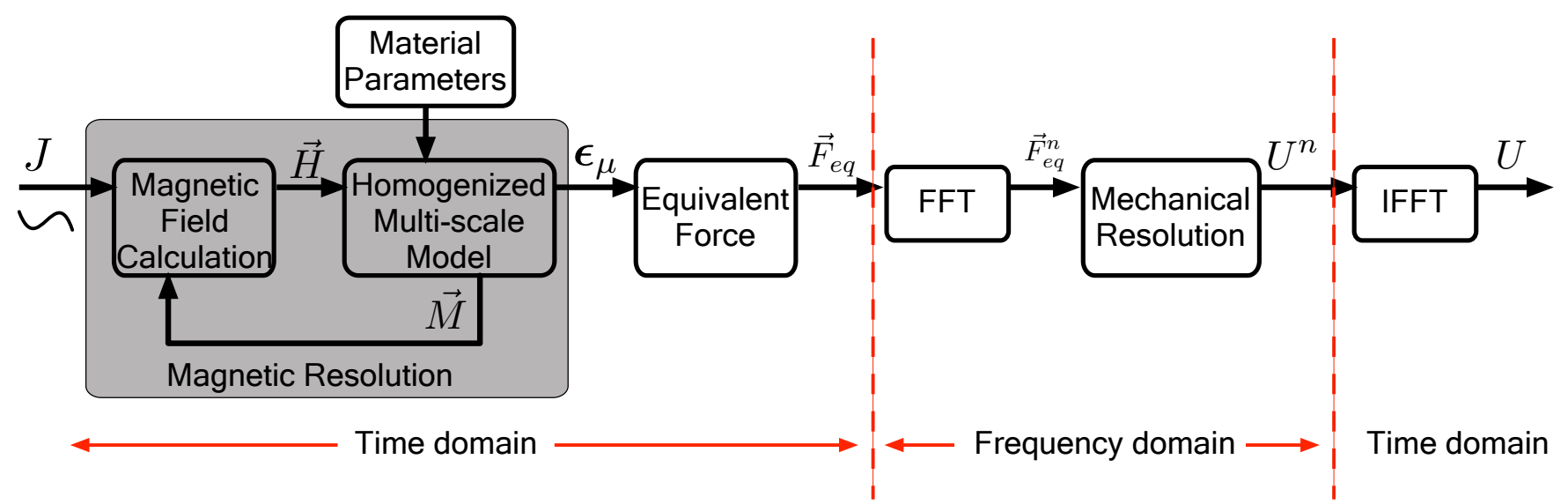

Fig. 3. Resolution strategy.

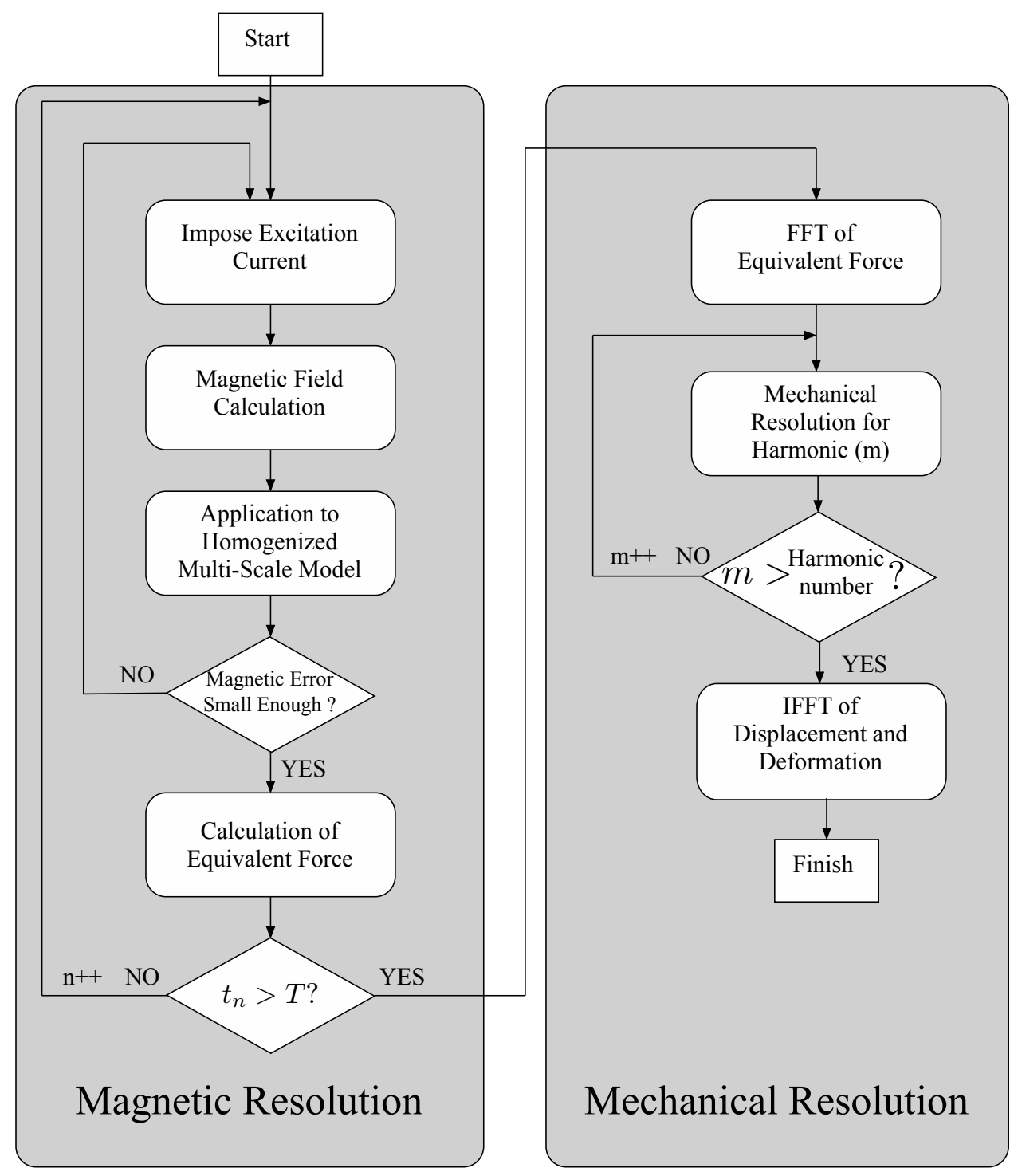

Fig. 4. Resolution algorithme.

\section{A. First Step: Magnetic Resolution}

At first, the magnetic problem is solved under static assumptions. Considering scalar potential formulation, magnetic field $\vec{H}$ is divided into two parts (32). One is the gradient of magnetic scalar 
potential $\Omega$. The other is the electrical vector potential $\vec{T}$, considered as the source term, which helps to impose excitation current in the coils (30).

$$
\begin{gathered}
\vec{H}=\vec{T}+\overrightarrow{\operatorname{grad}}(\Omega) \\
\operatorname{rot}(\vec{T})=\vec{J} \\
\operatorname{div}(\vec{B})=0 \\
\vec{B}=\mu_{0}(\vec{H}+\vec{M})
\end{gathered}
$$

The boundary condition would be $\vec{H}_{n}=0$, which set to zero the normal magnetic field far from the transformer. Therefor, the weak form of this problem discretized by finite elements leads to the nonlinear system given by equation (33). Its resolution gives the magnetic scalar potential $\Omega$ that leads to the calculation of magnetic field $\vec{H}$.

$$
[S][\Omega]=[T]
$$

Fig.5 shows the basic strategy of magnetic problem resolution, considering the previously defined homogenization technique. Two simplified MSM (one per each layer family) are integrated in the resolution chain using $\vec{H}$ as input, magnetization $\vec{M}_{1}, \vec{M}_{2}$ and free strain $\boldsymbol{\epsilon}_{\mu 1}, \boldsymbol{\epsilon}_{\mu 2}$ as output. An iterative resolution process with a modified fixed-point algorithm [33] is then required, due to the non-linearity of magnetic behavior. The new homogenized magnetization $\vec{M}$ is introduced as an input of magnetic resolution for the next iteration until convergence. The free homogenized magnetostrictive strain $\epsilon_{\mu}$ is calculated, using the $\epsilon_{\mu 1}$ and $\epsilon_{\mu 2}$ output (eq.20 and 28).

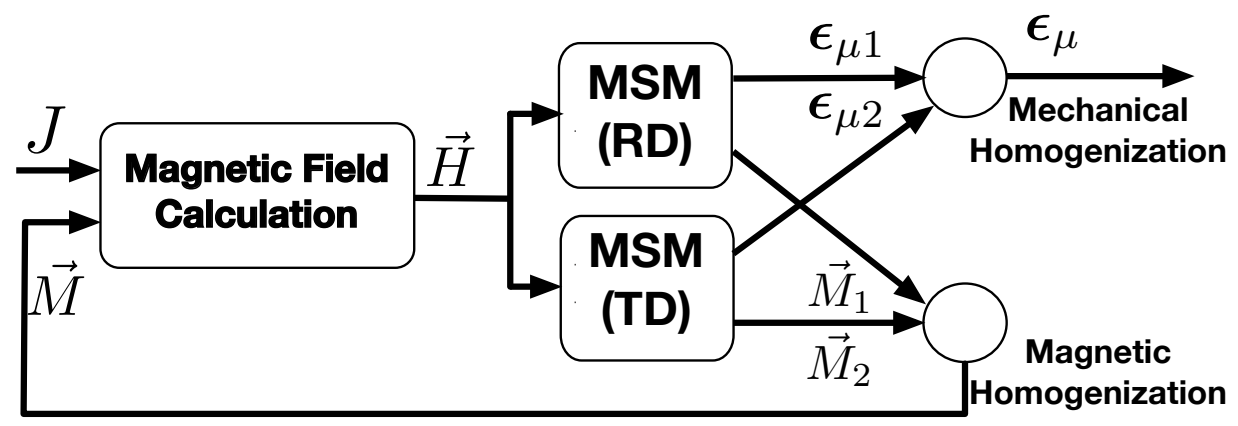

Fig. 5. Magnetic resolution.

\section{B. Second Step: Equivalent Force Calculation}

The second step consists in the calculation of an equivalent force field $\vec{F}_{e q}$ over a current period to obtain the mechanical loading of a pure vibrational problem. The mechanical constitutive law is written in the framework of linear elasticity (34), with $\mathbb{C}$ the stiffness tensor. The 2D plane stress assumption leads to a change of stiffness tensor expression comparing to $3 \mathrm{D}$ condition. A $3 \times 3$ stiffness tensor expression as function of $E$ and $\nu$ is given in equation (35). Stress $\hat{\sigma}$ and elastic strain $\hat{\epsilon}_{e}$ associated vectors are given in (36).

$$
\boldsymbol{\sigma}=\mathbb{C}: \boldsymbol{\epsilon}_{e}
$$




$$
\begin{gathered}
\mathbb{C}_{3 \times 3}=\left(\begin{array}{ccc}
\frac{E}{1-\nu^{2}} & \frac{\nu E}{1-\nu^{2}} & 0 \\
\frac{\nu E}{1-\nu^{2}} & \frac{E}{1-\nu^{2}} & 0 \\
0 & 0 & \frac{E}{1+\nu}
\end{array}\right) \quad \text { so that: } \quad \hat{\sigma}=\mathbb{C}_{3 \times 3} . \hat{\epsilon}_{e} \\
\hat{\sigma}=\left(\begin{array}{c}
\sigma_{X X} \\
\sigma_{Y Y} \\
\sqrt{2} \sigma_{X Y}
\end{array}\right) \quad \hat{\epsilon}_{e}=\left(\begin{array}{c}
\epsilon_{e X X} \\
\epsilon_{e Y Y} \\
\sqrt{2} \epsilon_{e X Y}
\end{array}\right) \quad \text { and } \quad \epsilon_{e Z Z}=-\frac{\nu}{1-\nu}\left(\epsilon_{e X X}+\epsilon_{e Y Y}\right)
\end{gathered}
$$

Considering volume forces $\vec{f}_{\text {ext }}$, the balance equation leads to:

$$
\overrightarrow{\operatorname{div} \sigma}+\vec{f}_{\text {ext }}=\rho \frac{\partial^{2} \vec{u}}{\partial t^{2}}
$$

Using the decomposition of total strain into elastic and magnetostrictive strain, Hooke's law is expressed as follows:

$$
\boldsymbol{\sigma}=\mathbb{C}:\left(\epsilon-\epsilon_{\mu}\right)
$$

Equation (37) then becomes:

$$
\overrightarrow{\operatorname{div}} \tilde{\boldsymbol{\sigma}}+\vec{f}_{e q}+\vec{f}_{e x t}=\rho \frac{\partial^{2} \vec{u}}{\partial t^{2}}
$$

with:

$$
\tilde{\boldsymbol{\sigma}}=\mathbb{C}: \boldsymbol{\epsilon} \quad \text { and } \quad \vec{f}_{\text {eq }}=-\overrightarrow{\operatorname{div}}\left(\mathbb{C}: \boldsymbol{\epsilon}_{\mu}\right)
$$

The magneto-elastic is transformed into a pure elastic problem with total and elastic deformation the strain tensor $\epsilon$ of a material submitted to a equivalent force density $\vec{f}_{e q}$. In the variational form, these expressions bring about nodal forces as following [34]:

$$
\vec{F}_{e q}=-\int_{\Omega}\left(\overrightarrow{g r a d}_{s}(v) \cdot \mathbb{C}: \boldsymbol{\epsilon}_{\mu}\right) \mathrm{d} \Omega
$$

Where $v$ is the test function. Nodal forces are used next as loading for the mechanical resolution. A force profile over the time $\vec{F}_{e q}(t)$ is obtained for each node.

\section{Third Step: Modal Resolution of the Mechanical Problem and Harmonics}

Once the equivalent force over time is calculated, a FFT of equivalent force is performed. The mechanical problem is solved for each harmonic of pulsation $\omega^{n}(42)$.

$$
\overrightarrow{d i v} \tilde{\boldsymbol{\sigma}}^{n}+\vec{f}_{e q}^{n}+\vec{f}_{e x t}^{n}=-\rho\left(\omega^{n}\right)^{2} \vec{u}^{n}
$$

Where $\vec{f}_{e q}^{n}$, the density of the equivalent force of $n^{\text {th }}$ harmonic, is obtained by dividing the equivalent nodal force with the surface associated to the corresponding node (dual cell). $\rho$ is the density and $\vec{u}^{n}$ the displacement field of the $n_{t h}$ harmonic. After finite element discretization, the weak form of equation (42) can be written as:

$$
\left([K]-\left(\omega^{n}\right)^{2}[M]\right)\left[U^{n}\right]=\left[F_{e q}^{n}\right]+\left[F_{e x t}^{n}\right]
$$

Where $[M]$ is the mass matrix, and $[K]$ is an stiffness corresponding matrix. By adding the damping coefficient of the material $\beta$, equation(43) becomes:

$$
\left([K]+2 j \beta \omega^{n}[K]-\left(\omega^{n}\right)^{2}[M]\right)\left[U^{n}\right]=\left[F_{e q}^{n}\right]+\left[F_{e x t}^{n}\right]
$$


Displacement solution of each harmonic is obtained by solving equation (44). The time domain solution is obtained by the inverse Fourier transform:

$$
u_{i}(t)=\sum_{n=1}^{N} U_{i}^{n} \exp \left(j \omega^{n} t\right)
$$

The time discretization $\Delta t$ over a period $T$ gives the maximal number of harmonics that are considered in the calculation: $\max (N)=T / 2 \Delta t-1$. Moreover it must be underlined that the constitutive behavior of material leads to an initial homogeneous deformation in the material even without electrical loading. The actual displacement field is the difference between the displacement with loading and the one without:

$$
u_{i}(t)^{\text {actual }}=u_{i}(t)^{\text {loaded }}-u_{i}(t)^{\text {unloaded }}
$$

Moreover a modal analysis can be implemented by solving the mechanical equation without applied force and damping:

$$
[K][U]-\omega^{2}[M][U]=[0]
$$

$[M]$ and $[K]$ are respectively the mass and stiffness corresponding matrix. The solving allows the calculation of the main vibration modes of the transformer structure.

\section{APPLICATION TO A GRAIN-ORIENTED SILICON-IRON ALLOY TRANSFORMER}

\section{A. Validation of simplified multi-scale model}

To validate the multi-scale model and the total chain of estimation of the noise emission, an anisotropic magnetic material is needed. In certain direction, the magnetostrictive strains are small and in others are strong. Grain-oriented (GO) silicon-iron alloy is one of the most popular soft ferromagnetic materials, which exhibits the so-called GOSS texture $(\{110\}<001>$ along rolling direction). In a previous study [6], it has been shown that GO FeSi carries strong anisotropy behaviors in both magnetic and magnetostrictive aspects. With the studied material, a $0.27 \mathrm{~mm}$ thick grain-oriented FeSi sheet, measurements of magnetization and magnetostrictive strain along RD and TD are compared with our simplified MSM (physical constants used for simplified MSM are shown in Table I).

TABLE I

PHYSICAL CONSTANTS USED FOR SIMPLIFIED MSM.

\begin{tabular}{|c|c|c|c|c|c|c|}
\hline Param. & $M_{s}$ & $K_{1}$ & $\lambda_{100} ; \lambda_{111}$ & $C$ & $N_{x x} ; N_{y y} ; N_{z z}$ & $A_{s}$ \\
\hline \hline Value & $1.61 \times 10^{6}$ & 38 & $23.5 ;-4.5$ & 300 & $0 ; 0 ; 1$ & $20 \times 10^{-3}$ \\
\hline Unit & $\mathrm{A} / \mathrm{m}$ & $\mathrm{kJ} . \mathrm{m}^{-3}$ & $\mathrm{ppm}$ & $\mathrm{J}^{-\mathrm{m}^{-3}}$ & - & - \\
\hline
\end{tabular}

Definition of specific $\mathbb{P}$ matrix associated to the material. We choose $\mathbb{P}$ for cubic material as defined by equation (4). The $\mathrm{Q}$ matrix is defined by:

$$
\mathbf{Q}_{I}=\left(\begin{array}{ccc}
1 & 0 & 0 \\
0 & \sqrt{2} / 2 & \sqrt{2} / 2 \\
0 & -\sqrt{2} / 2 & \sqrt{2} / 2
\end{array}\right) \quad \mathbf{Q}_{E}=\left(\begin{array}{ccc}
\sqrt{2} / 2 & 0 & \sqrt{2} / 2 \\
0 & 1 & 0 \\
-\sqrt{2} / 2 & 0 & \sqrt{2} / 2
\end{array}\right)
$$

Fig. 6 shows a strong anisotropic magnetic behavior for GO FeSi. Along RD, the magnetization reached is about $40 \%$ higher than that along TD. This is because of the crystallographic texture of the material, $\mathrm{RD}$ corresponds to a $\langle 100\rangle$ direction (easy axis) and TD to a $\langle 110\rangle$. Longitudinal and transversal strain measurements are shown in Fig.7 and Fig.8. When a magnetic field is applied along RD, an ultralow magnetostriction is highlighted. And when magnetic field is applied along TD, the longitudinal 


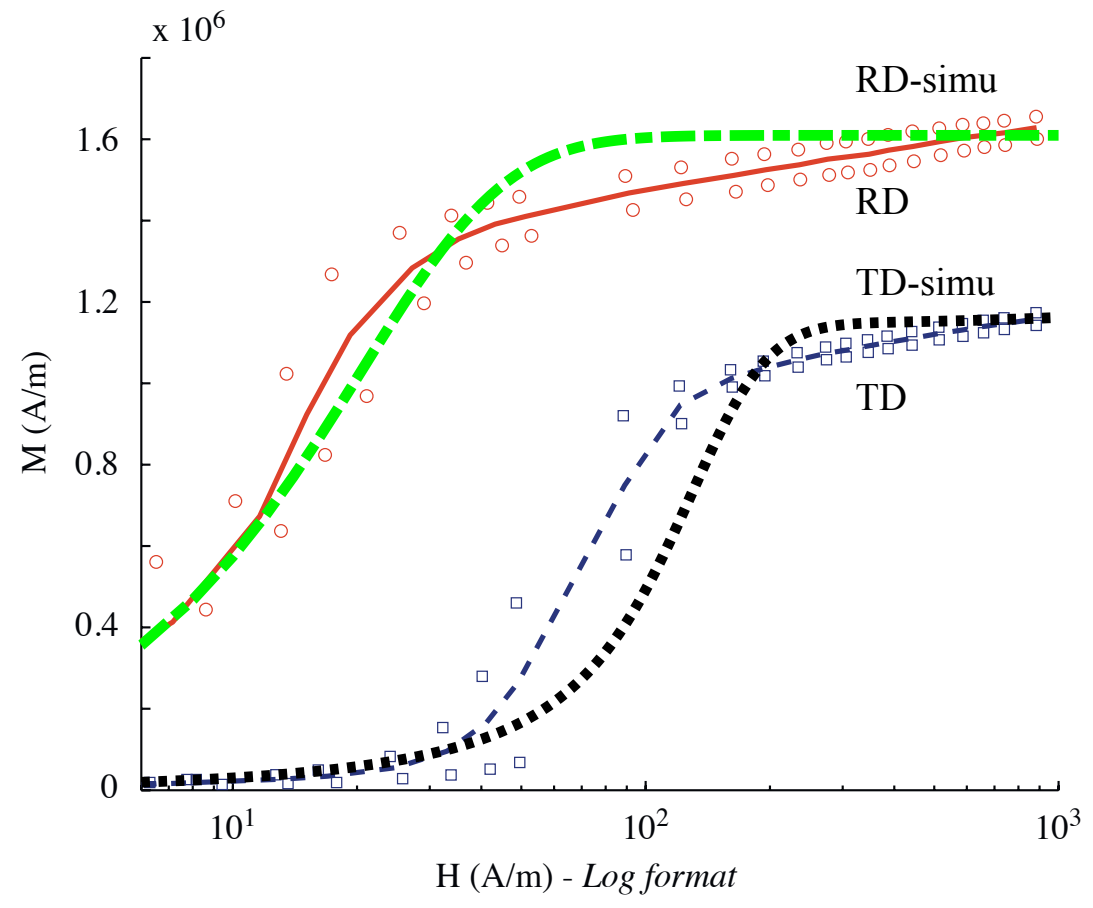

Fig. 6. Reserved for Comparison of simplified MSM to experimental measurement [6] of the anhysteretic magnetization curves along RD (red) and TD (blue).

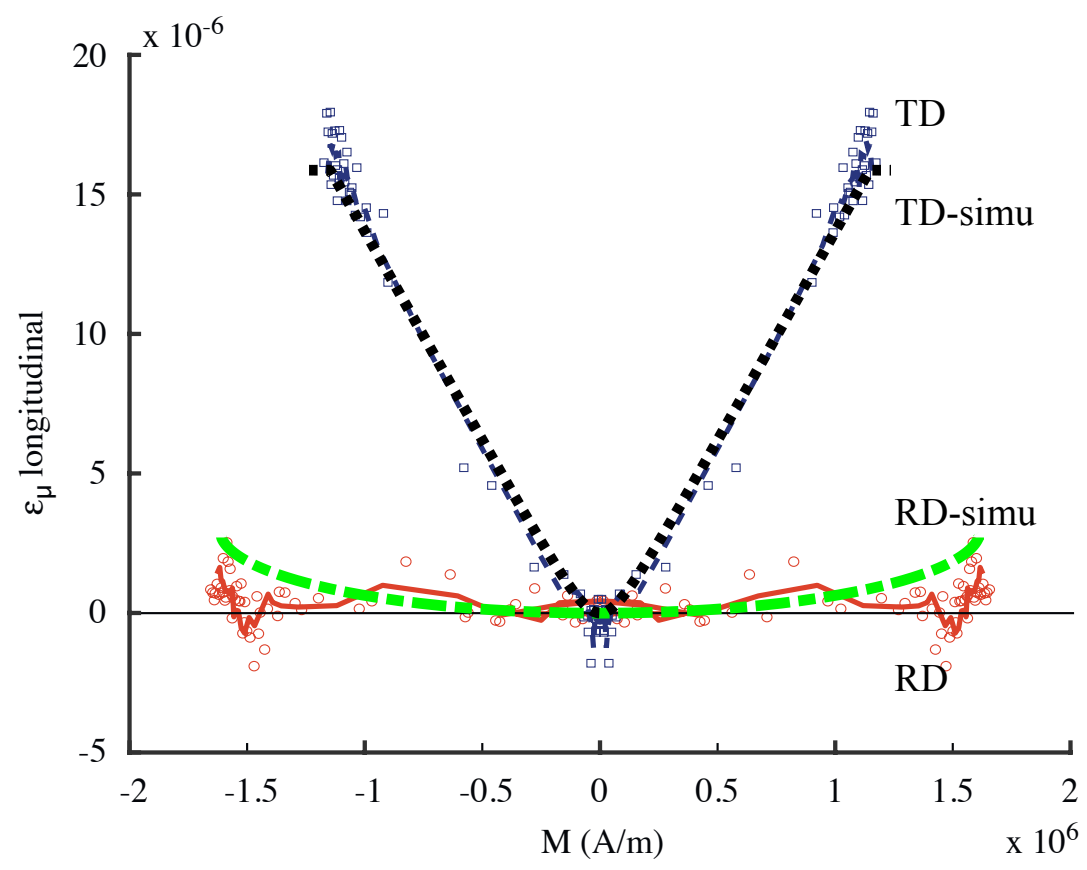

Fig. 7. Reserved for Comparison of simplified MSM to experimental measurement [6] of the anhysteretic longitudinal magnetostriction along RD (red) and TD (bleu).

magnetostriction strain reaches $18 \times 10^{-6}$. This anisotropy is due to the oriented grain texture, which increases the presence probability of the magnetic domain in certain direction (RD).

Results obtained with the simplified MSM for the considered material GO FeSi show a good agreement with the experimental results. 


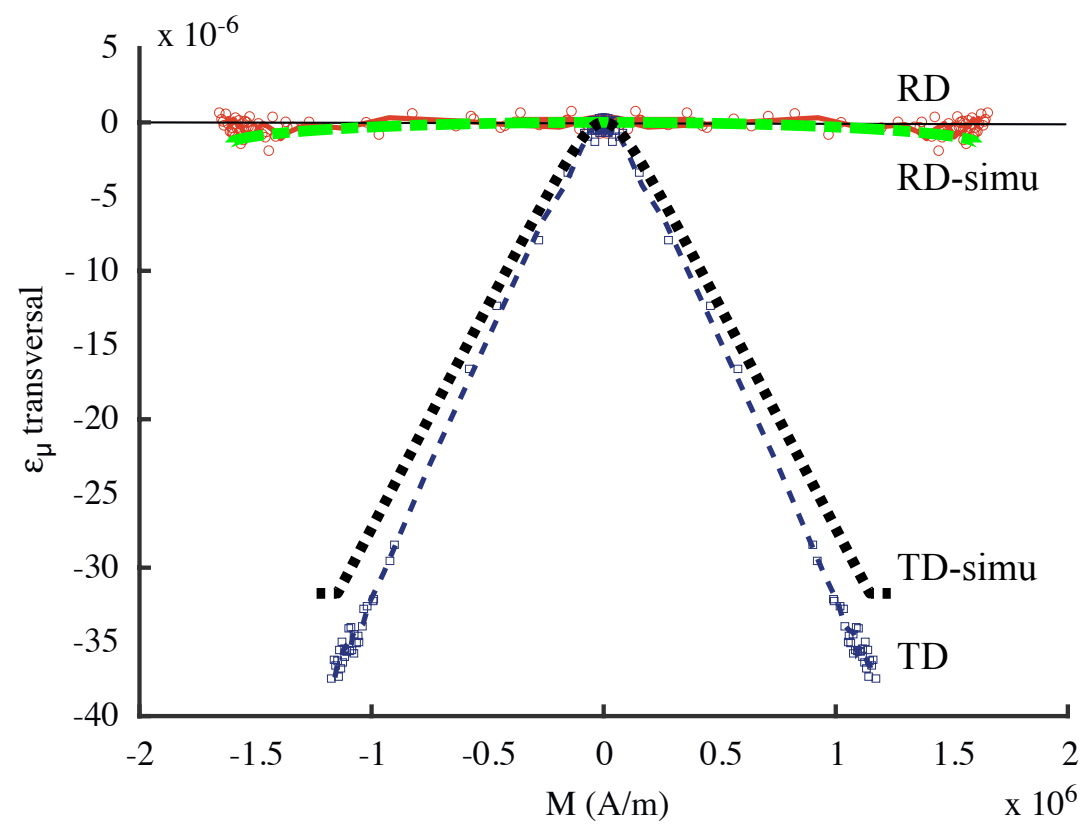

Fig. 8. Reserved for Comparison of simplified MSM to experimental measurement [6] of the anhysteretic transversal magnetostriction along $\mathrm{RD}$ (red) and TD (blue).

B. Multi-layer transformer modeling in $2 \mathrm{D}$ using $\mathrm{GO} \mathrm{FeSi}$

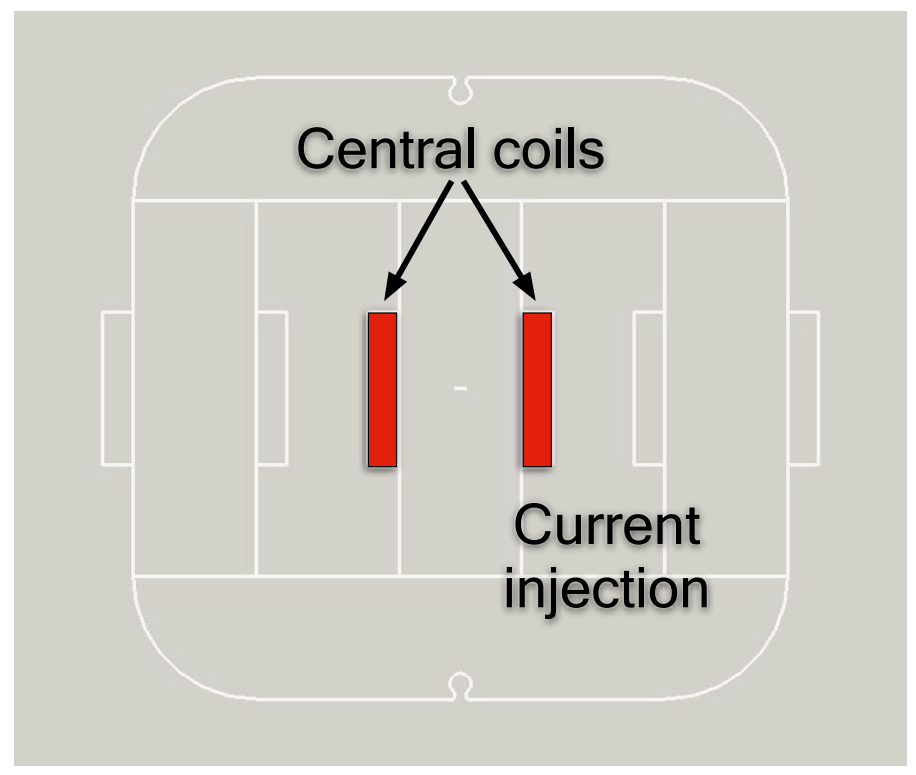

Fig. 9. Current injection.

For the purpose of simplification, it is chosen to excite only the central coil (Figure. 9) with a sinusoidal source expressed by discrete-time (49).

$$
I(n)=I_{\max } \cos \left(\frac{2 \pi n}{N}\right)
$$

Where $I_{\max }$ is amplitude of the the current, $\mathrm{N}$ is the total number of steps in one period, and $n$ represents the time index integer sequence $0,1,2$, etc. Sectional surface fraction $\left(f_{1}\right.$ and $\left.f_{2}\right)$ of different regions are set the same as in section II to simulate a three-layer transformer. This is in the aim of comparison with the 
measurements carried out on our three-layer transformer prototype (Cf. Section V-C). The reference point of the transformer is forced fixed using Dirichlet boundary condition, so that relative displacements of the other parts are calculated (reference point is not at the center to avoid the coils when doing experimental measurements). The triangular non-uniform mesh is refined in the regions where saturation appears.

For simplification considerations, mechanical behavior is considered elastic, isotropic and homogeneous (extension to anisotropic elastic behavior is foreseen).

TABLE II

PHYSICAL CONSTANTS AND NUMERICAL SETTINGS USED FOR TRANSFORMER DEFORMATION MODELING IN 2 D.

\begin{tabular}{|c|c|c|c|c|c|c|}
\hline Param. & $I_{\max }$ & $f$ & $\beta$ & $N$ & $E$ & $\nu$ \\
\hline \hline Value & 200 & 400 & 0.02 & 40 & 210 & 0.3 \\
\hline Unit & Amper-turn & $\mathrm{Hz}$ & - & - & $\mathrm{GPa}$ & - \\
\hline
\end{tabular}

The force profile over the time $\vec{F}_{e q}(t)$ obtained at one node of the mesh is illustrated in Figure 10

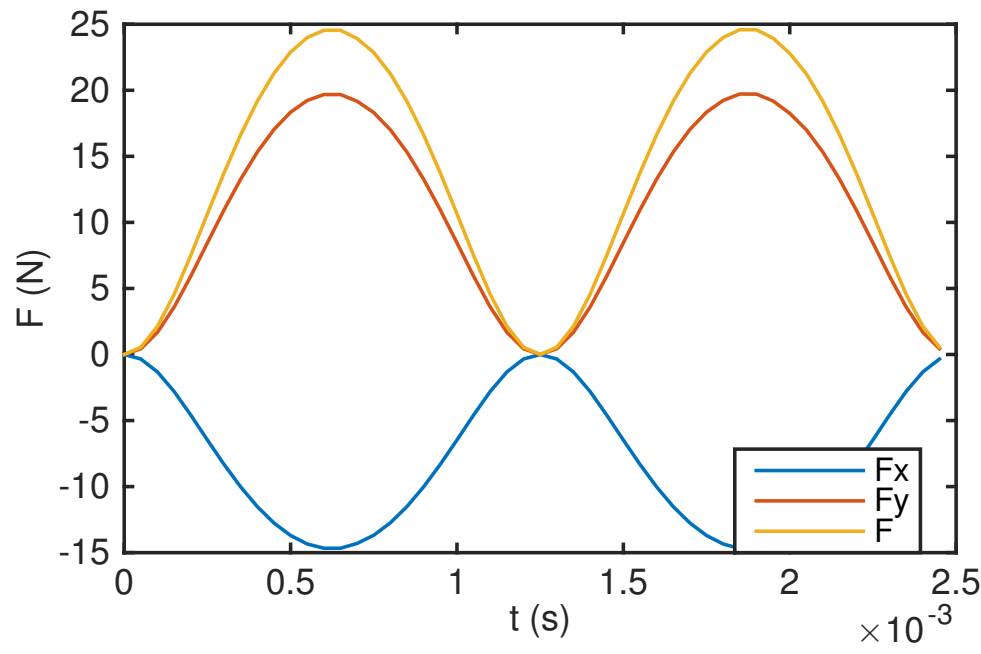

Fig. 10. Profile of equivalent force $\vec{F}_{e q}$ components and norm at one node (point 2 in Fig.15) over one period of current.

Fig.11 shows the flux distribution. Fig.12 shows the distribution of the nodal equivalent force of magnetostriction. Based on virtual work, a calculation of magnetic forces (without magnetostriction) is also carried out, using exactly the same material properties, homogenisation techniques and calculation strategy. The latter is aimed to verify its small effect compared to magnetostriction. From Fig.12, it is clear that the magnetic force is negligible with respect to forces induced by magnetostriction. Fig.13 and 14 show respectively the components of total strain and displacement at the moment when excitation current reaches its maximum. The area with the strongest strain is exactly where the magnetic field is aligned with TD, which is in accordance with the anisotropic material behaviors. Related displacements are created due to these magnetostrictive strains. A scale factor of $10 e 5$ is used for better visibility of the tiny displacements.

\section{Experimental measurements and comparisons with modeling}

To measure particular points' displacements in transformer core, several accelerometers are placed to record the acceleration over several periods of excitation. Then it's followed by two integrations to get a 


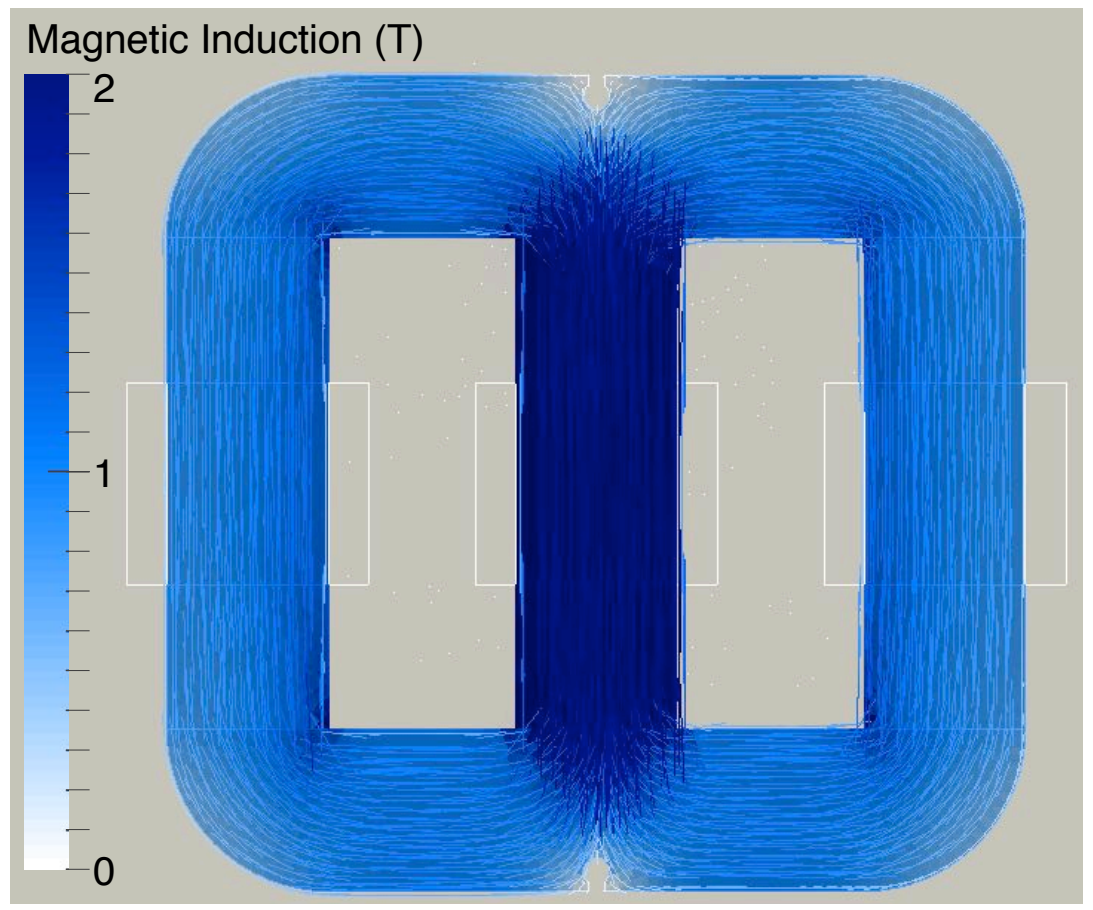

Fig. 11. Distribution of magnetic induction.

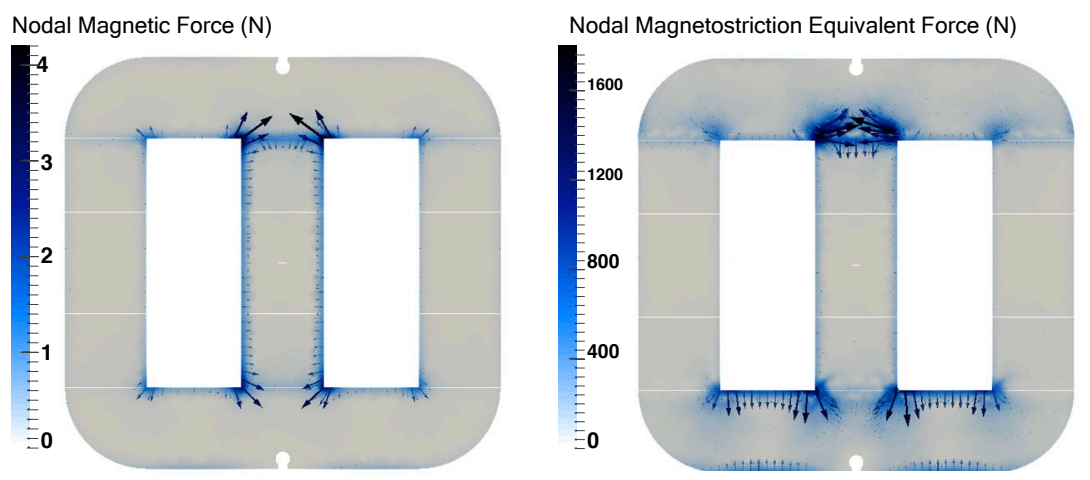

Fig. 12. Distribution of nodal magnetic force (left) and nodal magnetostrictive equivalent force (right).

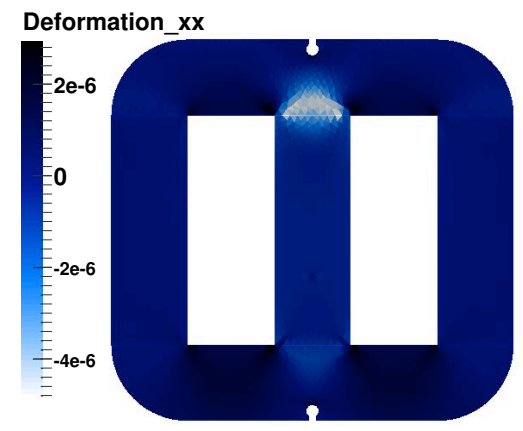

(a)

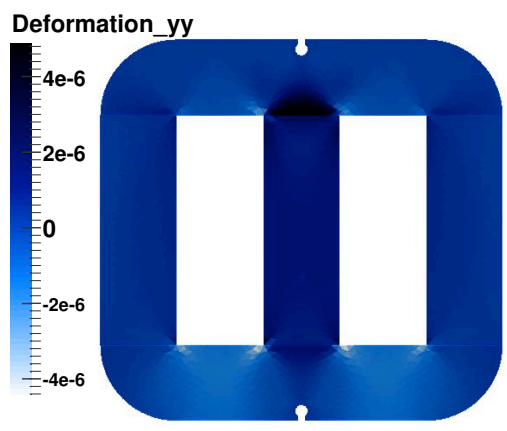

(b)

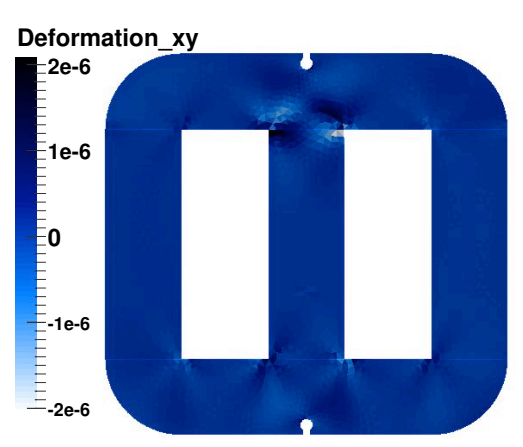

(c)

Fig. 13. Core total deformation fields at $I=I_{\max }=5 \mathrm{~A}$ with 40 turns.

displacement. Several regions with relatively large displacement, according to the modeling, are chosen to install accelerometers (Fig.15). 


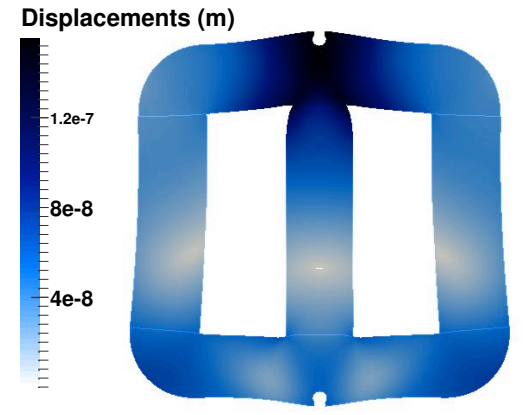

(a)

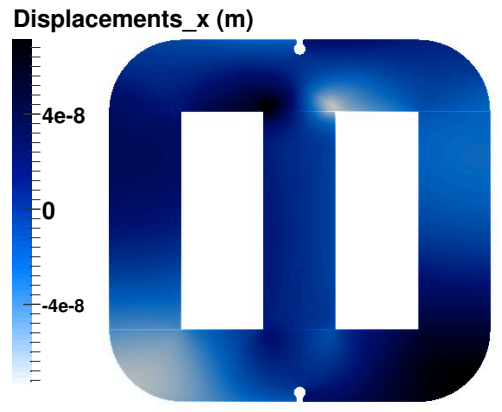

(b)

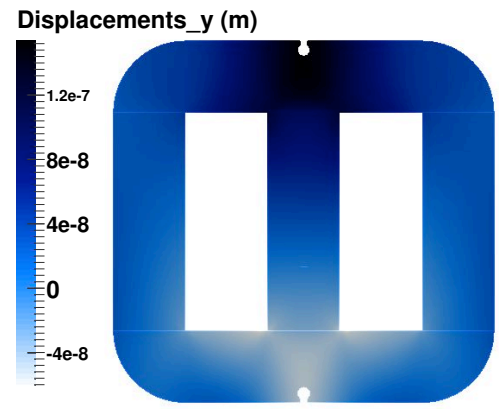

(c)

Fig. 14. Core displacement fields at $I=I_{\max }=5 A$ with 40 turns.

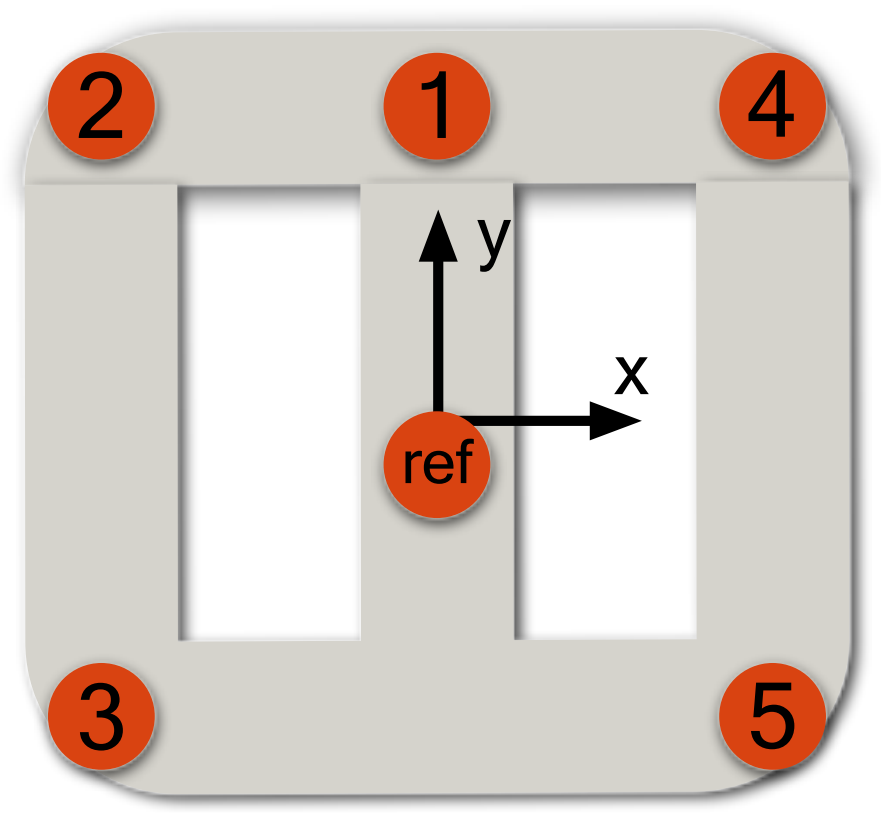

Fig. 15. Displacement measured points.

The center of the transformer is set as a reference point, so as to measure the relative displacement compared to the center. An average can be made among the measuring points (points 2,4 and 3,5) thanks to the symmetry of the geometry with y-axis (There is no symmetry with respect to the $\mathrm{x}$-axis because of the different RD of each sheet).

To measure the acceleration, a three-layer transformer prototype is hung up with two ropes, shown as Fig.16, for mainly two reasons: -To eliminate the external out-plane stress. -To eliminate the perturbation of rigid body movement in the post-processing with a low-pass filter. A sinusoidal current, with amplitude $5 A$, is injected in the central coil at $400 \mathrm{~Hz}$. Numerical simulation is carried out with the same condition as experiment (excitation, geometry, volume fraction and the reference point). Vibration frequency is two times higher than current frequency, because the positive and negative magnetization create the same strain. This permits an application of a low-pass filter at $700 \mathrm{~Hz}$ to purify the measured accelerations and then displacements. The same test is carried out five times for averaging. Displacement within one period of the injection current is shown in Fig.17, 18, 19 (notice that the three figures are not in the same scale), with full line representing measured displacement and dotted line representing simulated displacement. For better visibility, the displacement along direction $\mathrm{X}$ and $\mathrm{Y}$ is presented separately in red and blue.

This numerical calculation has successfully predicted the trend of transformer deformation, with a good 


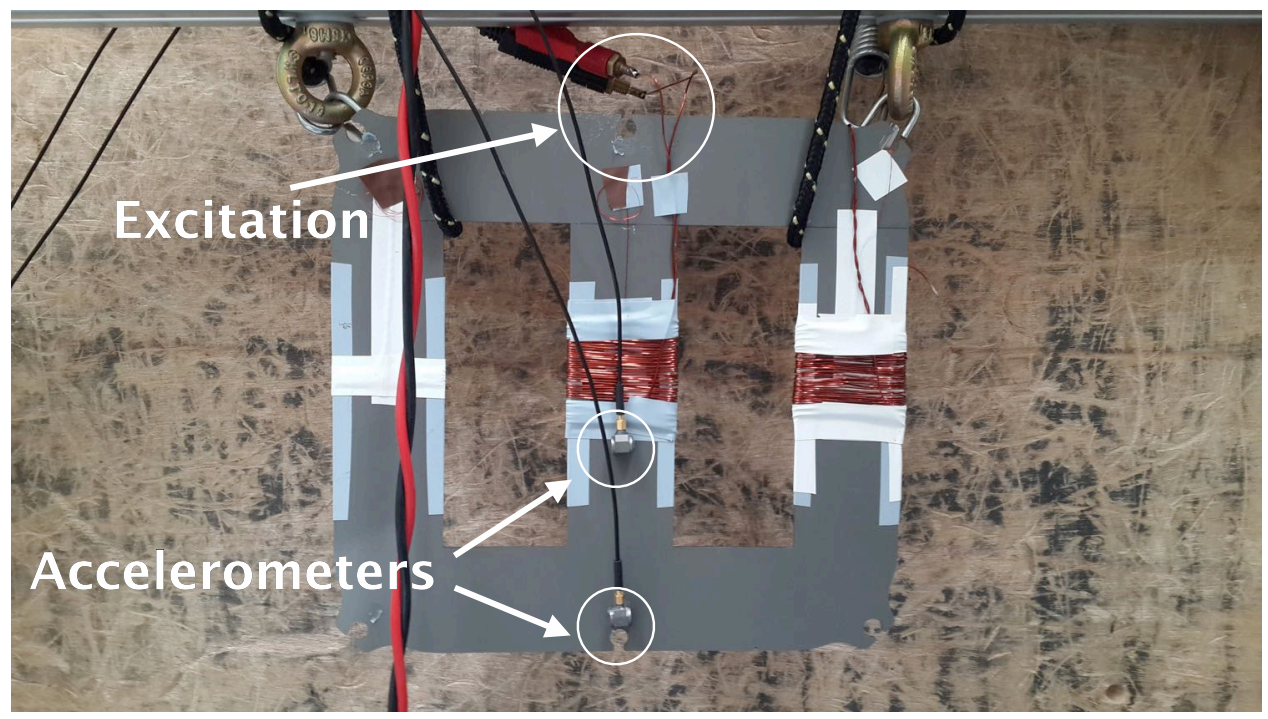

Fig. 16. Experimental set-up for displacement measurement.

accordance between measurements and simulation such as : displacement period, direction and order of magnitude.

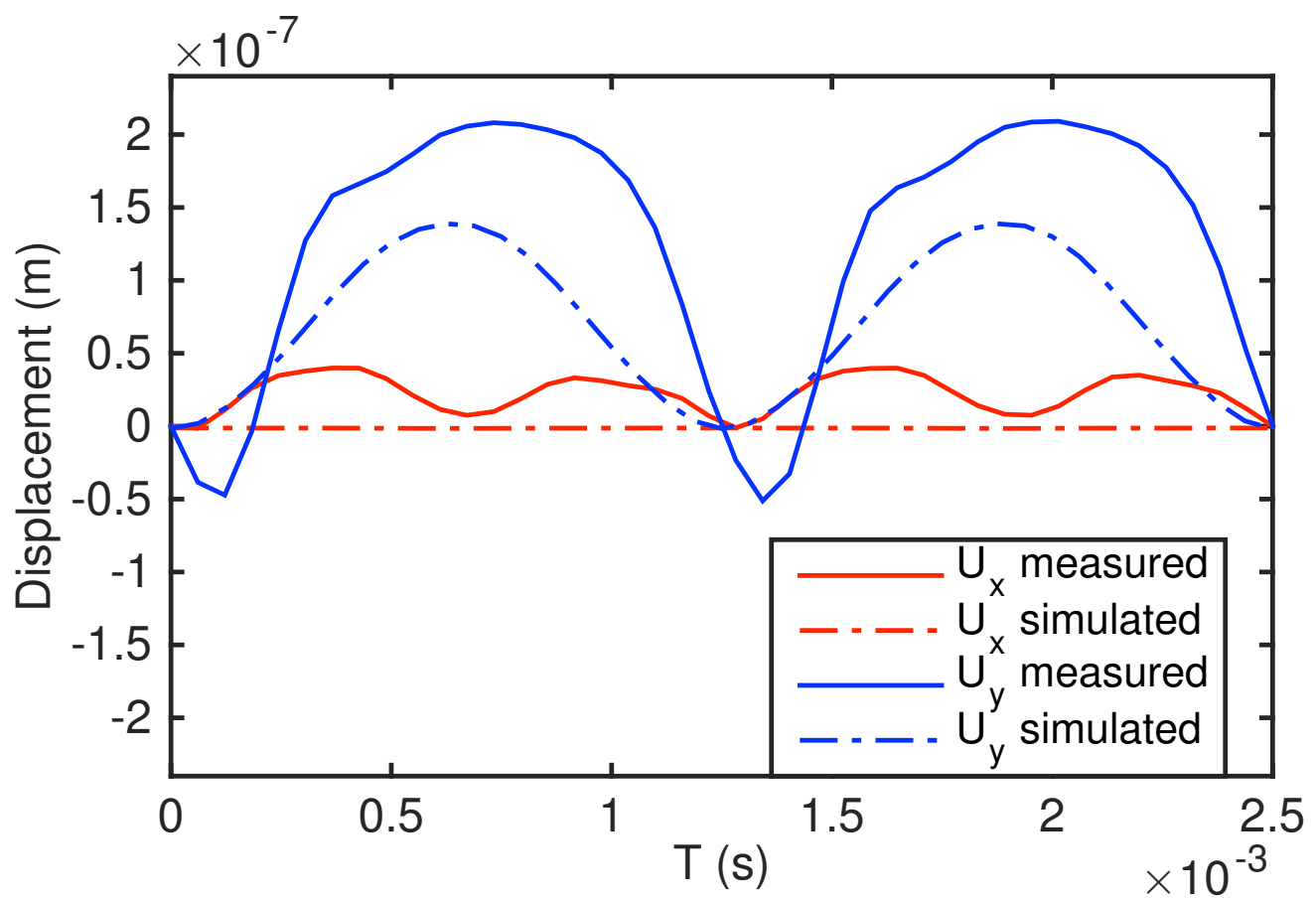

Fig. 17. Displacement comparison between modeling and measurement at point 1 .

The numerical model accurately predicts displacements along direction X, except for point 1 (Fig.17) with a measured doubled frequency $(1600 \mathrm{~Hz})$ which should be zero considering the symmetry. This may be due to the non-perfect symmetry of the transformer. Even slightly unbalanced forces may create large displacements at the resonance frequency. A modal analysis is carried out for the entire structure ('E+I') and also for sheets 'I' and 'E' separately. The main vibration modes of the structure are presented in Table III. Mode 2 corresponding to frequency $1515 \mathrm{~Hz}$ for sheet ' $E$ ' is plotted in Fig.20. This mode could 


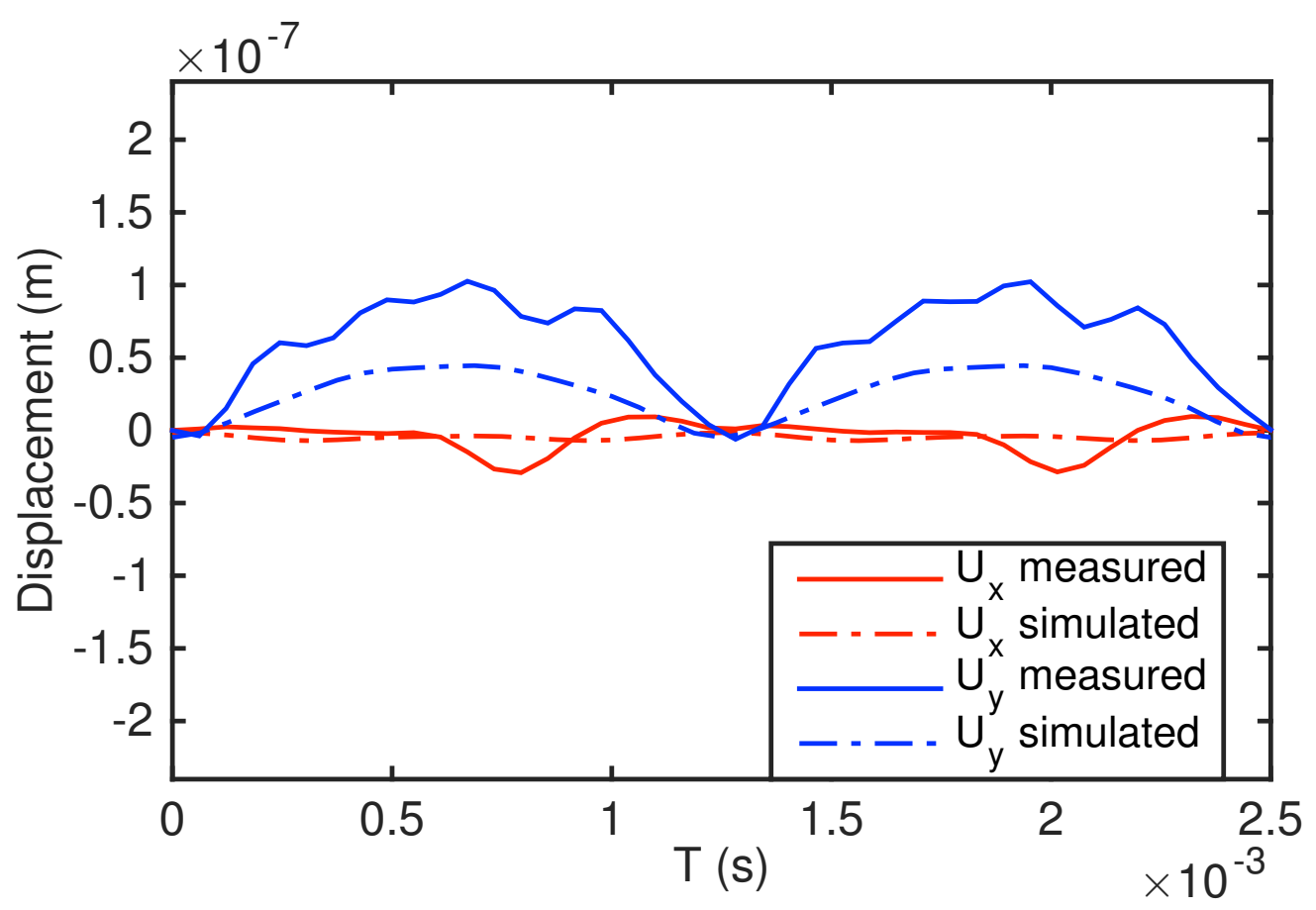

Fig. 18. Displacement comparison between modeling and measurement at point 2 .

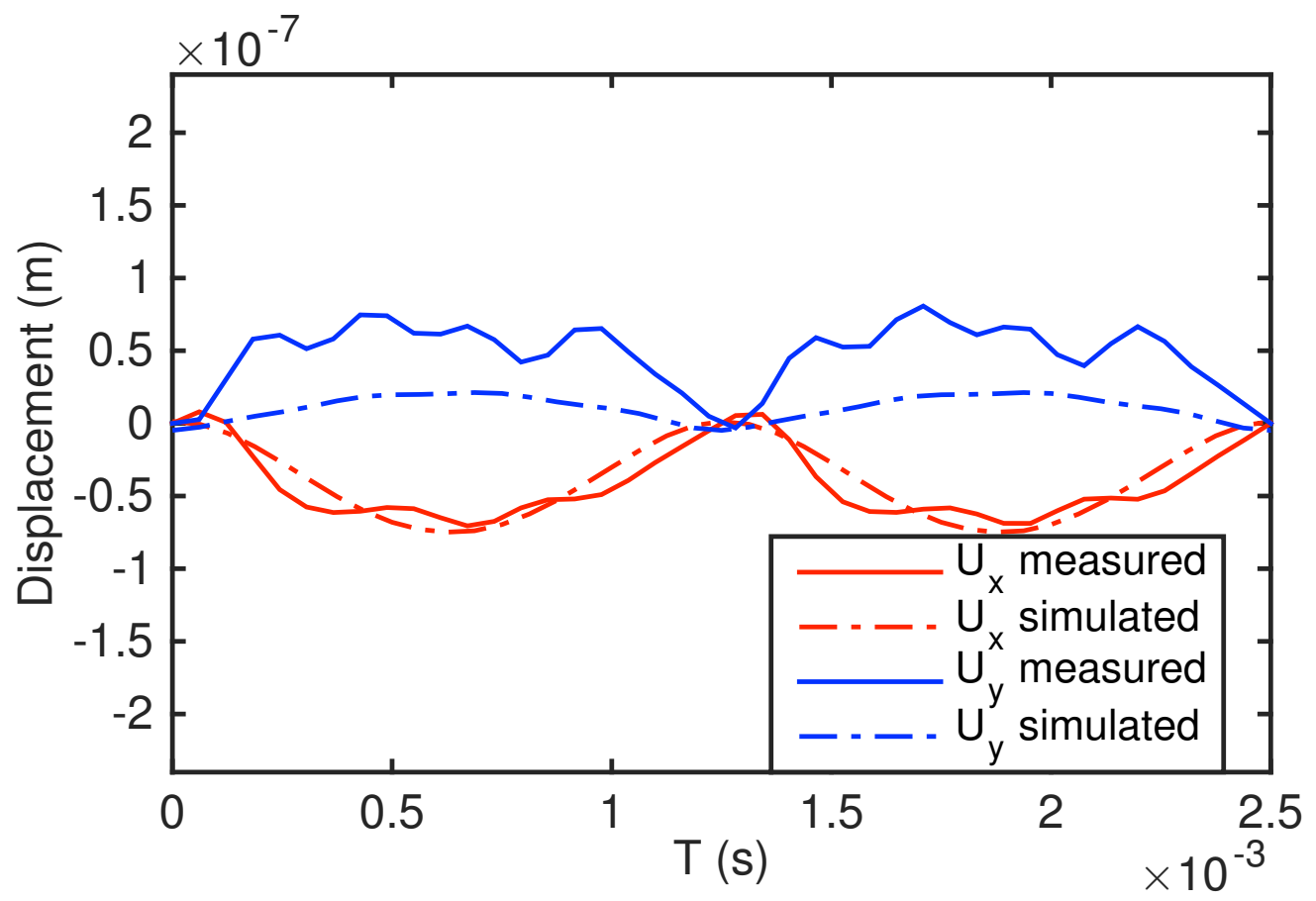

Fig. 19. Displacement comparison between modeling and measurement at point 3 .

explain the measured amplitude at the point 1.

Measured displacements along direction $\mathrm{Y}$ is larger than predicted results. This can be explained by the existence of air-gap, not considered in model, that weakens the mechanic holdings in direction Y. To verify this explanation, air-gap has to be added in the model. 
TABLE III

RESONANCE MODE OF STRUCTURE 'E', 'I', 'E+I'

\begin{tabular}{cccc} 
mode number & $\mathrm{E}$ & $\mathrm{I}$ & $\mathrm{E}+\mathrm{I}$ \\
\hline \hline 1 & $1007 \mathrm{~Hz}$ & $4839 \mathrm{~Hz}$ & $3717 \mathrm{~Hz}$ \\
2 & $1515 \mathrm{~Hz}$ & $12707 \mathrm{~Hz}$ & $5457 \mathrm{~Hz}$ \\
3 & $2984 \mathrm{~Hz}$ & $13271 \mathrm{~Hz}$ & $5867 \mathrm{~Hz}$ \\
4 & $4406 \mathrm{~Hz}$ & $21264 \mathrm{~Hz}$ & $5928 \mathrm{~Hz}$
\end{tabular}

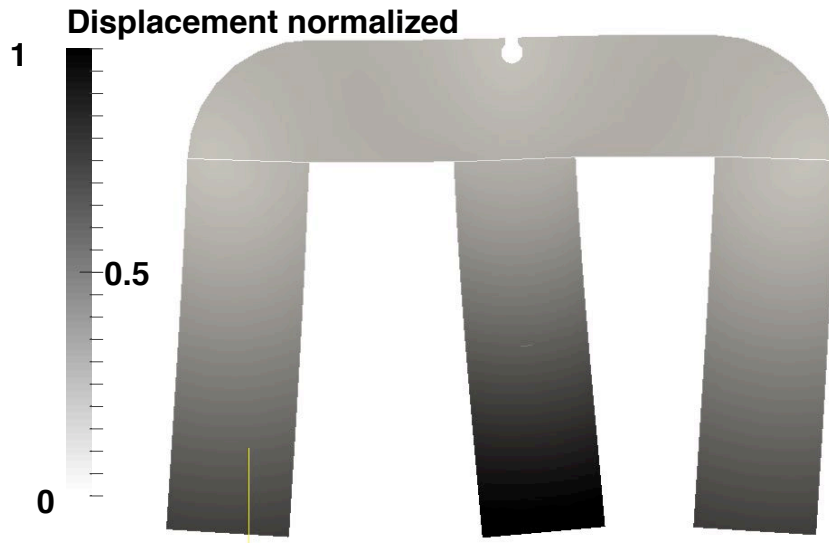

Fig. 20. Resonance mode for sheet 'E' with frequency $1515 \mathrm{~Hz}$.

\section{CONCLUSION}

The proposed model, taking into account the anisotropy of the material and surface effect, allows the calculation of the 2D strain field of a multi-layer electrical transformer. The good accordance with experimental tests confirms the reliability of this model. The model is highly dependent on the experimental characterization of the material presented in this paper. Such study shows a feasibility of geometrical optimization of the transformer design in order to reduce the strain level and consequently noise level. A final coupling between strain field and acoustic noise is still missing at this step. Futher step would be to achieve an optimization of both material and design to reduce the noise emission.

\section{REFERENCES}

[1] M. Ertl and H. Landes, "Investigation of load noise generation of large power transformer by means of coupled 3D FEM analysis," COMPEL, vol. 26, no. 3, pp. $788-799,2007$.

[2] Y.-H. Chang, C.-H. Hsu, H.-L. Chu, and C.-P. Tseng, "Magnetomechanical Vibrations of Three-Phase Three-Leg Transformer With Different Amorphous-Cored Structures," Magnetics, IEEE Transactions on, vol. 47, no. 10, pp. 2780-2783, Oct. 2011.

[3] C.-H. Hsu, Y.-H. Chang, C.-Y. Lee, C.-S. Yao, Y.-L. He, H.-L. Chu, C.-W. Chang, and W.-S. Chan, "Effects of magnetomechanical vibrations and bending stresses on three-phase three-leg transformers with amorphous cores," Journal of Applied Physics, vol. 111, no. $7,2012$.

[4] E. Du Trémolet de Lacheisserie, Magnetostriction. CRC Press, 1993.

[5] J. L. Coulomb, "No Title," IEEE Transactions on Magnetics, no. 19, p. 2514, 1983.

[6] O. Hubert and L. Daniel, "Multiscale modeling of the magneto-mechanical behavior of grain-oriented silicon steels," Journal of Magnetism and Magnetic Materials, vol. 320, no. 7, pp. 1412-1422, Apr. 2008.

[7] K. Fonteyn, A. Belahcen, R. Kouhia, P. Rasilo, and A. Arkkio, "FEM for Directly Coupled Magneto-Mechanical Phenomena in Electrical Machines," Magnetics, IEEE Transactions on, vol. 46, no. 8, pp. 2923-2926, Aug. 2010.

[8] A. Belahcen, "Magnetoelastic Coupling in Rotating Electrical Machines," Magnetics, IEEE Transactions on, vol. 41, no. 5, pp. 1624$1627,2005$.

[9] B. Weiser and H. Pfützner, "Relevance of magnetostatic forces for transformer core vibrations," Le Journal de Physique IV, vol. 8, no. 1 998, pp. 8-11, 1998.

[10] Y. Wang, J. Pan, and M. Jin, "Finite Element Modelling of the Vibration of a Power Transformer," Proceedings of ACOUSTICS, no. 34, pp. 1-7, 2011. 
[11] M. B. Balehosur, P. Marketos, A. J. Moses, and J. N. Vincent, "Packet-to-Packet Variation of Flux Density in a Three-Phase, Three-Limb Power Transformer Core," IEEE Transactions on Magnetics, vol. 46, no. 2, pp. 642-645, Feb. 2010.

[12] R. Penin, J.-p. Lecointe, G. Parent, J.-f. Brudny, S. Member, and T. Belgrand, "Grain Oriented Steel Rings for an Experimental Comparison of Relative Magnetostriction and Maxwell s Forces Effects," IEEE Transactions on Industrial Electronics, vol. 61, no. 8, pp. 1-8, 2014.

[13] H. Pfützner, E. Mulasalihovic, H. Yamaguchi, D. Sabic, G. Shilyashki, and F. Hofbauer, "Rotational magnetization in transformer cores - A review," IEEE Transactions on Magnetics, vol. 47, no. 11, pp. 4523-4533, 2011.

[14] G. Shilyashki, H. Pfutzner, J. Anger, K. Gramm, F. Hofbauer, V. Galabov, and E. Mulasalihovic, "Magnetostriction of transformer core steel considering rotational magnetization," IEEE Transactions on Magnetics, vol. 50, no. 1, 2014.

[15] S. Somkun, A. J. Moses, P. I. Anderson, and P. Klimczyk, "Magnetostriction anisotropy and rotational magnetostriction of a nonoriented electrical steel," IEEE Transactions on Magnetics, vol. 46, no. 2, pp. 302-305, 2010.

[16] L. Daniel, O. Hubert, and M. Rekik, "A simplified 3D constitutive law for magneto-mechanical behavior," IEEE Transactions on Magnetics, 2015.

[17] A. Baghel, A. Gupta, K. Chwastek, and S. Kulkarni, "Comprehensive modelling of dynamic hysteresis loops in the rolling and transverse directions for transformer laminations," Physica B: Condensed Matter, vol. 462, pp. 86-92, 2015.

[18] a. Ebrahimi and a. J. Moses, "Correlation between normal flux transfer from lamination to lamination during the magnetization process with static domain structures in grain oriented 3\% silicon iron," Journal of Applied Physics, vol. 70, p. 6265, 1991.

[19] Y. Gao, K. Muramatsu, M. J. Hatim, and M. Nagata, "The effect of laminated structure on coupled magnetic field and mechanical analyses of iron core and its homogenization technique," in IEEE Transactions on Magnetics, vol. 47, no. 5, 2011 , pp. 1358-1361.

[20] N. Hihat, E. Napieralska-Juszczak, J. P. Lecointe, J. K. Sykulski, and K. Komeza, "Equivalent Permeability of Step-Lap Joints of Transformer Cores: Computational and Experimental Considerations," Magnetics, IEEE Transactions ..., vol. 47, no. 1, pp. 244-251, Jan. 2000.

[21] M. Javorski, G. Cepon, J. Slavic, and M. Boltezar, "A generalized magnetostrictive-forces approach to the computation of the magnetostriction-induced vibration of laminated steel structures," IEEE Transactions on Magnetics, vol. 49, no. 11, pp. 5446-5453, 2013.

[22] C. Krell, N. Baumgartinger, and G. Krismanic, "Relevance of multidirectional magnetostriction for the noise generation of transformer cores," Journal of Magnetism and Magnetic Materials, vol. 216, pp. 634-636, 2000.

[23] B. Weiser, H. Pfützner, J. Anger, H. Pfutzner, and J. Anger, "Relevance of magnetostriction and forces for the generation of audible noise of transformer cores," in IEEE Transactions on Magnetics, vol. 36, no. 5, 2000, pp. 3759-3777.

[24] G. Shilyashki, H. Pfutzner, P. Hamberger, M. Aigner, and M. Palkovits, "A Tangential Induction Sensor for 3D-Analyses of Peripheral Flux Distributions in Transformer Cores," IEEE Transactions on Magnetics, vol. 9464, no. c, pp. 1-1, 2015.

[25] P. Witczak, "Magnetostriction Force Spectrum in Power Transformer," Electrical Machines (ICEM), 2014 International Conference, no. 3, pp. 2246-2251, 2014.

[26] Y. Wang and J. Pan, "Comparison of Mechanically and ElectricallyExcited Vibration Frequency Responses of a Small DistributionTransformer," IEEE Transactions on Power Delivery, vol. 8977, no. c, pp. 1-1, 2015.

[27] Z. Valkovic, "Effects of transformer core design on noise level," Le Journal de Physique IV, pp. 11-14, 1998.

[28] Z. Xian and Z. Pengcheng, "Numerical estimation and optimization of vibration noise due to magnetostriction and magnetic forces for laminated core structure," ... and Systems (ICEMS), ..., no. 51307120, pp. 1548-1551, 2014.

[29] L. Daniel, O. Hubert, N. Buiron, and R. Billardon, "Reversible magneto-elastic behavior: A multiscale approach," Journal of the Mechanics and Physics of Solids, vol. 56, no. 3, pp. 1018-1042, Mar. 2008.

[30] O. Hubert and L. Daniel, "Effect of plastic straining on magnetostriction of ferromagnetic polycrystals experiments and multiscale modeling," Journal of Magnetism and Magnetic Materials, vol. 304, pp. 489-491, 2006.

[31] N. Buiron, L. Hirsinger, and R. Billardon, "A multiscale model for magneto-elastic couplings," J. Phys. IV, no. 9, pp. 187-196, 1999.

[32] M. Liu, O. Hubert, X. Mininger, F. Bouillaut, and L. Bernard, "Calculation of Magnetostriction Induced Deformations in Grain Oriented and Non-Oriented Silicon Iron Transformer Cores Thanks to an Imposed Magnetic Flux Method," Proceedings of the Conference on the Computation of Electromagnetic Fields, 2015.

[33] F. Hantila, G. Preda, and M. Vasiliu, "Polarization method for static fields," Magnetics, IEEE Transactions on, vol. 36, no. 4, pp. 672-675, Jul. 2000.

[34] N. Galopin, X. Mininger, F. Bouillault, and L. Daniel, "Finite Element Modeling of Magnetoelectric Sensors," Magnetics, IEEE Transactions on, vol. 44, no. 6, pp. 834-837, Jun. 2008. 